\title{
COMPARISON OF DENOISING AlgorithMS FOR DEMOSACING LOW LIGHTING IMAGES USING CFA 2.0
}

\author{
Chiman Kwan and Jude Larkin \\ Applied Research, LLC, Rockville, Maryland, USA
}

\begin{abstract}
In modern digital cameras, the Bayer color filter array (CFA) has been widely used. It is also widely known as CFA 1.0. However, Bayer pattern is inferior to the red-green-blue-white (RGBW) pattern, which is also known as CFA 2.0, in low lighting conditions in which Poisson noise is present. It is well known that demosaicing algorithms cannot effectively deal with Poisson noise and additional denoising is needed in order to improve the image quality. In this paper, we propose to evaluate various conventional and deep learning based denoising algorithms for CFA 2.0 in low lighting conditions. We will also investigate the impact of the location of denoising, which refers to whether the denoising is done before or after a critical step of demosaicing. Extensive experiments show that some denoising algorithms can indeed improve the image quality in low lighting conditions. We also noticed that the location of denoising plays an important role in the overall demosaicing performance.
\end{abstract}

\section{KEYWORDS}

Bayer pattern, RGBW pattern, CFA 1.0, CFA 2.0, color filter array, demosaicing, denoising, pansharpening, deep learning

\section{INTRODUCTION}

Bayer pattern [1] was invented in the early 1980's and is still a very popular color filter array (CFA) for digital cameras. The Bayer pattern as shown in Figure 1(a) is also known as CFA 1.0 in the literature. Even for planetary explorations, NASA has adopted the Bayer pattern in the Mastcam imagers onboard the Mars rover Curiosity [2]-[5].

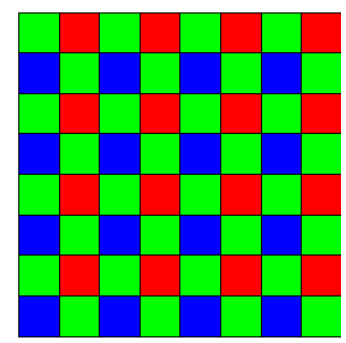

(a)

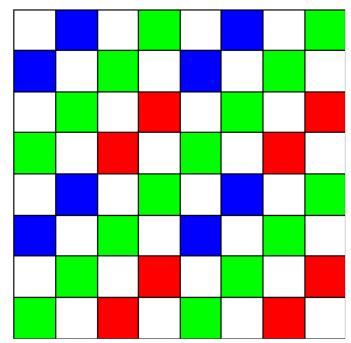

(b)

Figure 1. Two CFA patterns. (a) CFA 1.0; (b) CFA 2.0.

Aiming to improve the Bayer pattern in low lighting conditions, Kodak researchers [6,7] invented a red-green-blue-white (RGBW) CFA pattern, which is also known as CFA 2.0, as shown in 
Signal \& Image Processing: An International Journal (SIPIJ) Vol.11, No.5, October 2020

Figure 1(b). Half of the pixels in CFA 2.0 are white and the remaining pixels share the R, G, and $\mathrm{B}$ colors. Due to the presence of white pixels, the camera sensitivity is increased and hence the performance of CFA 2.0 in low lighting conditions should be better than CFA 1.0. Extensive experiments in [8] showed that CFA 2.0 is in indeed better than CFA 1.0 in low lighting conditions, where Poisson noise is dominant. Figure 2 shows a clean color image and two noisy images with different levels of Poisson noise. It can be seen that the noise can seriously affect the visual quality of the images. In low lighting conditions, demosaicing methods alone are not sufficient in suppressing the noise. Although there are some joint demosaicing and denoising algorithms such as [9] in the literature, those algorithms are tailored to only Gaussian noise. In an earlier paper [8], we developed new demosaicing algorithms for CFA 2.0. In the process, we also investigated the impact of denoising on the overall image quality. However, the denoising investigation in [8] was limited to only one method, the block matching in 3D (BM3D), even though the performance BM3D is reasonable.

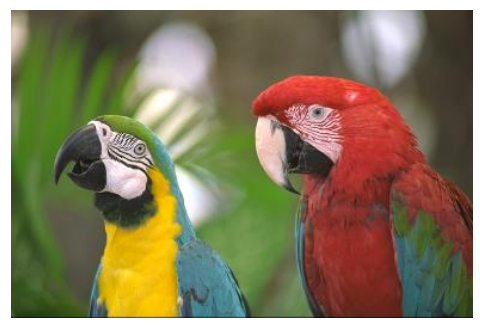

(a) Clean image

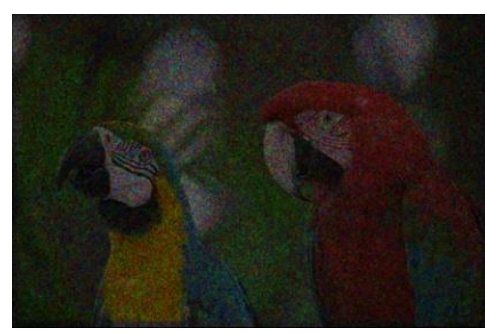

(b) $10 \mathrm{~dB}$ Noisy image

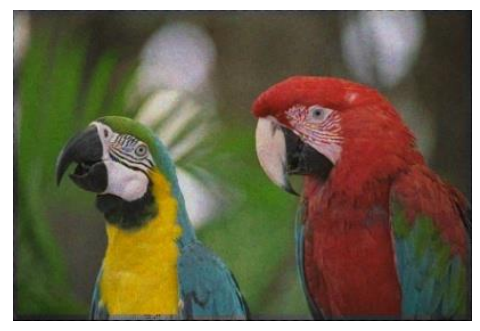

(c) $20 \mathrm{~dB}$ noisy image

Figure 2. Comparison of clean and noisy images with different levels of Poisson noise.

To the best of our knowledge, joint denoising and demosacing for CFA 2.0 is underdeveloped in the literature. In this paper, we will thoroughly investigate different algorithms in dealing with Poisson noise. We focus on CFA 2.0 because it was concluded in our earlier papers [8]10]-[12] that CFA 2.0 has better performance in low lighting conditions. Since only one denoising algorithm was used in [8], we would like to investigate how much performance we can further improve if we adopt other conventional and new denoising algorithms. In particular, we applied six conventional and one deep learning algorithms for suppressing Poisson noise. Two signal-tonoise (SNR) levels (10 dB and $20 \mathrm{~dB}$ ) of Poisson noise were introduced into clean Kodak images. Moreover, three denoising configurations were also investigated. This is because, in our earlier paper [8], we observed that the location of denoising can have very different overall performance in the final demosaiced images.

Our contributions are as follows. First, we thoroughly compared seven denoising algorithms for low lighting images. Some filters can improve the image quality quite significantly. Second, three denoising configurations were studied. One configuration works better than others. Third, we are the first team to carry out denoising and demosaicing studies for CFA 2.0.

The rest of this paper is organized as follows. Section 2 summarizes the methods, data, and performance metrics. In Section 3, we present the denoising results for two noisy conditions. Finally, we conclude the paper with a few remarks and future directions.

\section{Methods, Data, And Performance Metrics}

\subsection{Architecture}

Figure 3 shows the architecture of the proposed joint denoising and demosaicing system. Given an RGBW or CFA 2.0 image, we apply the Linear Directional Interpolation and Nonlocal 
Signal \& Image Processing: An International Journal (SIPIJ) Vol.11, No.5, October 2020

Adaptive Thresholding (LDI-NAT) [13] algorithm to demosaic a reduced resolution CFA 1.0 image. Parallel to this activity, the same LDI-NAT is applied to panchromatic image with $50 \%$ pixels missing to generate a full resolution illuminance image. We use the term panchromatic or illuminance interchangeably to represent the intensity image in this paper. After the above two steps, a denoising procedure is performed on both the panchromatic image and the reduced resolution color image. The denoised image is then going through a pansharpening process to generate the demosaiced image. Finally, another post-filtering is performed. It should be noted that denoising can also be done simultaneously before and after pansharpening and we call this option the hybrid denoising scheme.

Based on the above brief description, we can have three denoising configurations:

- Pre-denoising: This means that denoising is done before pansharpening starts. As shown in Figure 3, there are two places for pre-denoising: one for reduced resolution color image and one for the full resolution illuminance or panchromatic band.

- Post-Denoising: Here, denoising is done after the demosaiced image is obtained.

- Hybrid Denoising: This configuration basically includes both pre-denoising and postdenoising.

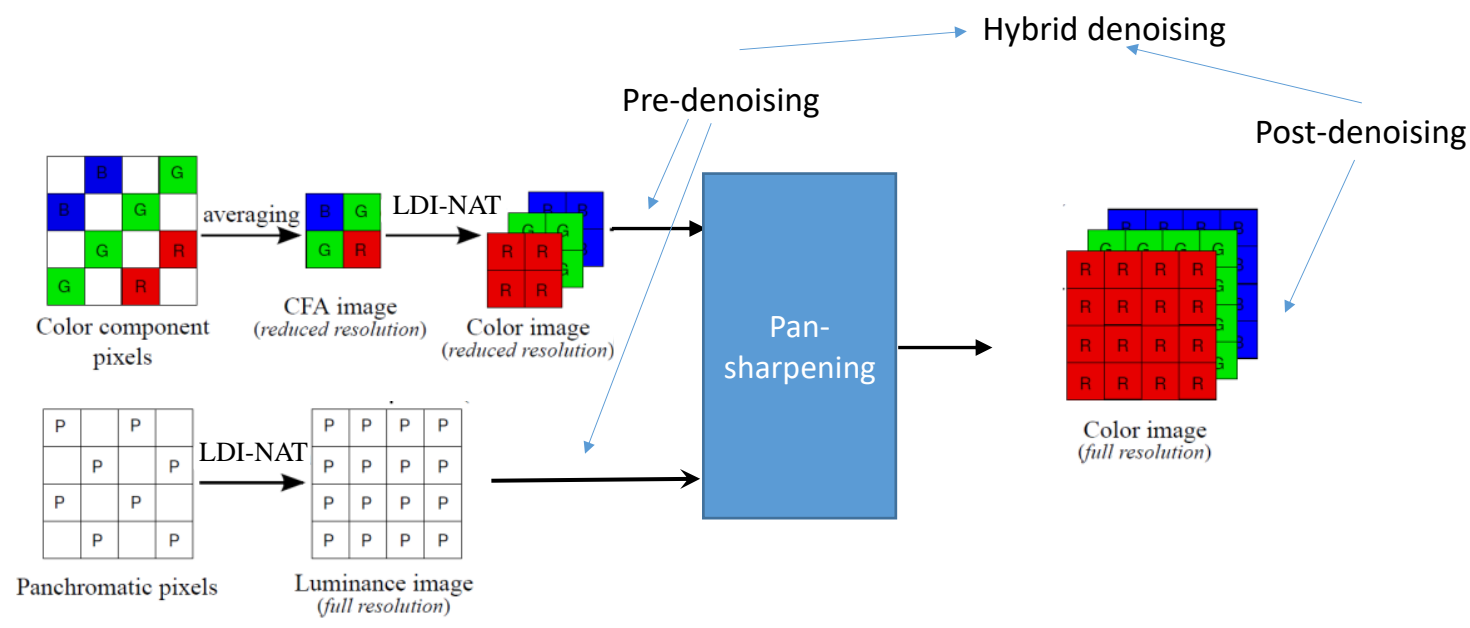

Figure 3. Architecture of joint denoising and demosaicing system for CFA 2.0.

\subsection{Denoising Methods}

Although there are many denoising methods in the literature, in this paper, we evaluated the following algorithms:

- Block Matching in 3 D (BM3D) [14]: This is a well-known denoising algorithm in the literature. The basic idea is to introduce exact unbiased inverses of the Anscombe and Generalized Anscombe transformations to deal with low-count (low photons) images. There are versions for Gaussian and Poisson noises. We used the version for Poisson and the codes can be found in [14].

- Wavelet [15]: The wavelet denoising consists of several steps. First, the input image is decomposed into several scales using discrete wavelet transform (DWT). Second, 
Signal \& Image Processing: An International Journal (SIPIJ) Vol.11, No.5, October 2020

thresholding is performed to the wavelet coefficients. Third, the denoising image is reconstructed from the thresholded DWT coefficients. We used the code in Matlab.

- Diffusion: According to [16], is a technique aiming at reducing image noise without removing significant parts of the image content. We used the Matlab codes [17], which does not specify whether the filter is suitable for Gaussian or other types of noise.

- Median Filter [18]: There are three variants of varying filter sizes $(3 \times 3,5 \times 5,7 \times 7)$. The reason for using median filters is because we observe that the noisy images have some resemblance to salt and pepper noise, which can be seen in those noisy images in Figure 2.

- FFDNet [19]: This is a deep learning based filtering algorithm. The first layer is a reversible downsampling operator which reshapes a noisy image into four downsampled sub-images. The second step involves the use of CNN for denoising. It has performed well on real images.

\subsection{Demosaicing Methods}

For CFA 2.0, there are not that many algorithms. In this paper, we adopted Linear Directional Interpolation and Nonlocal Adaptive Thresholding (LDI-NAT), which can be used for both demosaicing as well as interpolation [13]. It has good performance in our earlier studies [8]. We also used LDI-NAT in another earlier paper of ours [10]. As shown in Figure 3, LDI-NAT is used in two places: demosaicing the reduced resolution Bayer pattern and interpolating the panchromatic band.

In the paper [20] written by us, we proposed a pansharpening approach to demosaicing CFA 2.0. The missing pixels in the panchromatic band are interpolated. At the same time, the reduced resolution CFA is demosaiced. We then apply pansharpening to generate the full resolution color image. There are many pansharpening algorithms that can be used. Principal Component Analysis (PCA) [21], Smoothing Filter-based Intensity Modulation (SFIM) [22], Modulation Transfer Function Generalized Laplacian Pyramid (GLP) [23], MTF-GLP with High Pass Modulation (HPM) [24], Gram Schmidt (GS) [25], GS Adaptive (GSA) [26], Guided Filter PCA (GFPCA) [27], PRACS [28] and hybrid color mapping (HCM) [29]-[33] have been used in our experiments. The list is a representative, if not exhaustive, set of competitive pansharpening algorithms. Details of the above algorithms can be found in the corresponding papers and we omit the details in order to make our paper concise.

\subsection{Low Lighting Images}

We downloaded a benchmark data set (Kodak) from a website (http://r0k.us/graphics/kodak/) and selected 12 images, which are shown in Figure 4. It should be noted that this dataset is wellknown and has been used by many authors in the demosaicing community such as [34]-[38]. These clean images will be used as reference images for objective performance metrics generation. Moreover, they will be used for generating noisy images that emulate low lighting conditions. 
Signal \& Image Processing: An International Journal (SIPIJ) Vol.11, No.5, October 2020

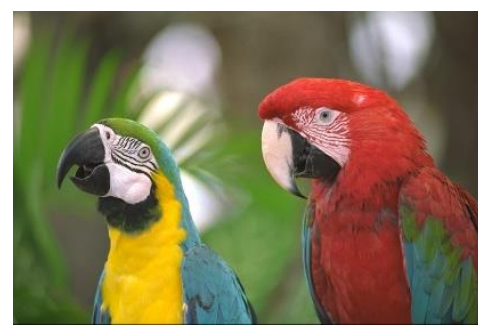

Image 1

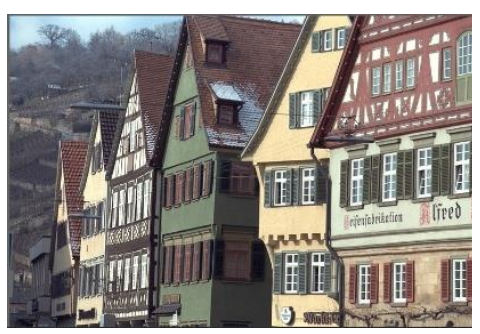

Image 4

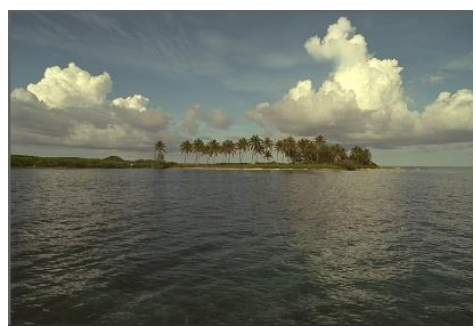

Image 7

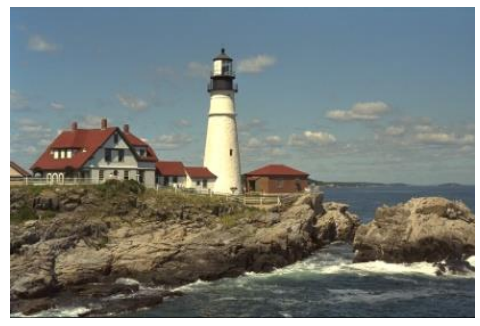

Image 10

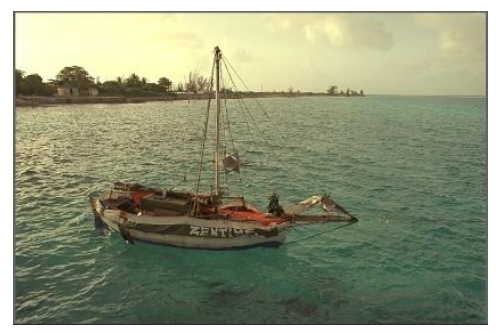

Image 2

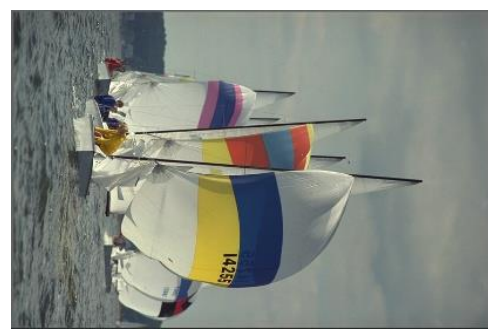

Image 5

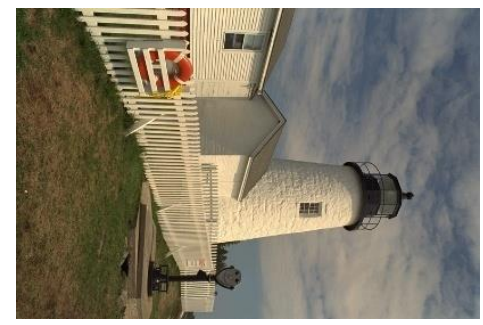

Image 8

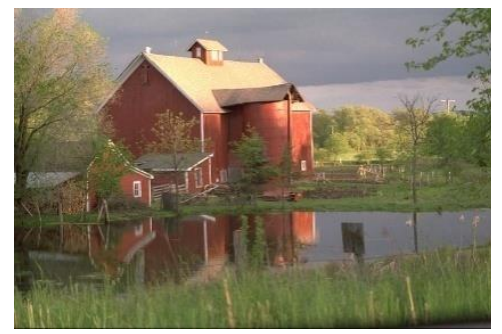

Image 11

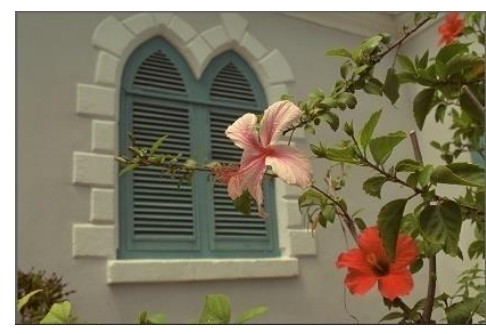

Image 3

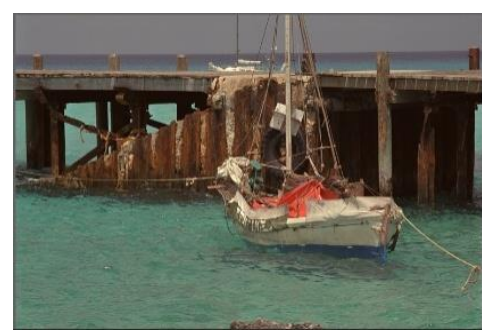

Image 6

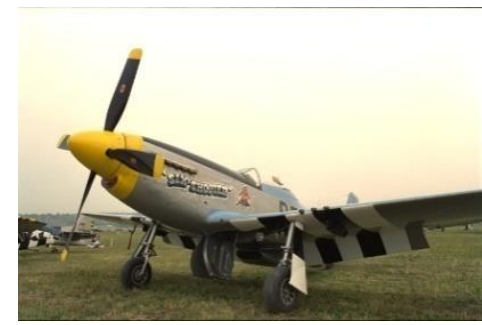

Image 9

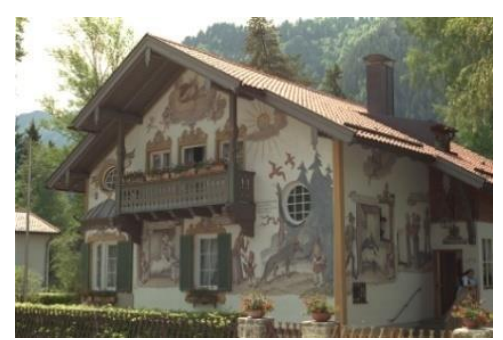

Image 12

Figure 4. Twelve clean images from the Kodak dataset.

The process of how we introduced Poisson noise is adapted from code written by Erez Posner (https://github.com/erezposner/Shot-Noise-Generator). Details can be found in our recent paper [10]. We include the Poisson noisy $10 \mathrm{~dB}$ and $20 \mathrm{~dB}$ images in Figure 5 and Figure 6, respectively. 
Signal \& Image Processing: An International Journal (SIPIJ) Vol.11, No.5, October 2020

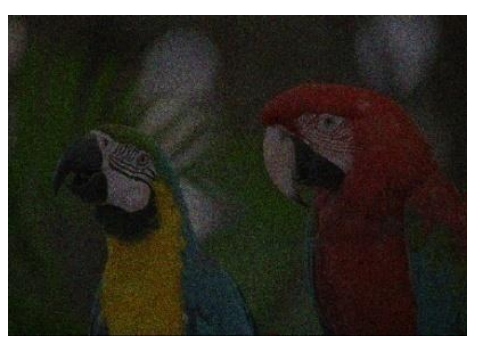

Image 1

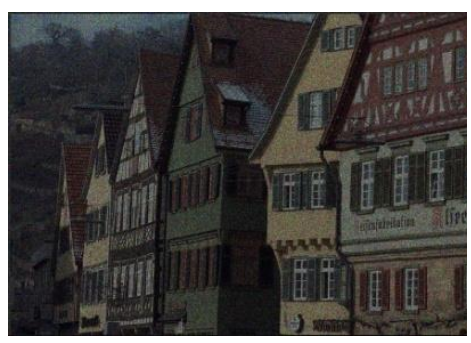

Image 4

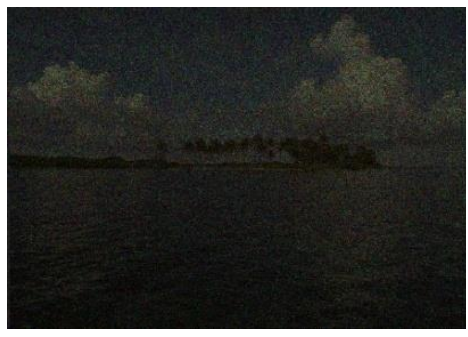

Image 7

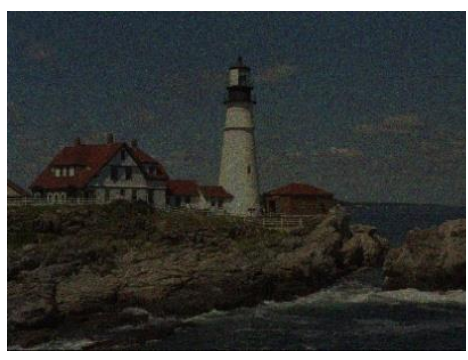

Image 10

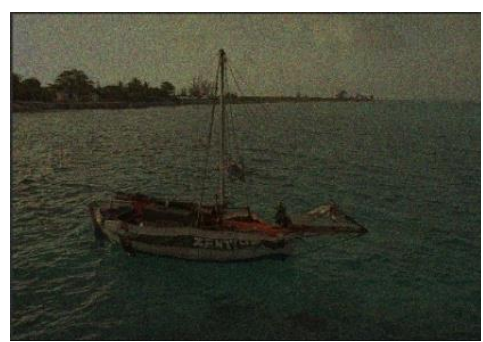

Image 2

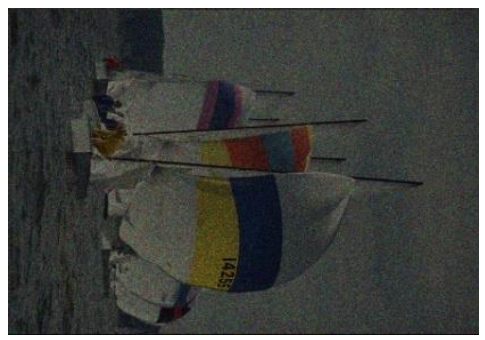

Image 5

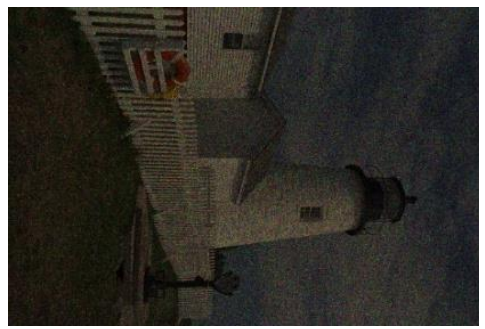

Image 8

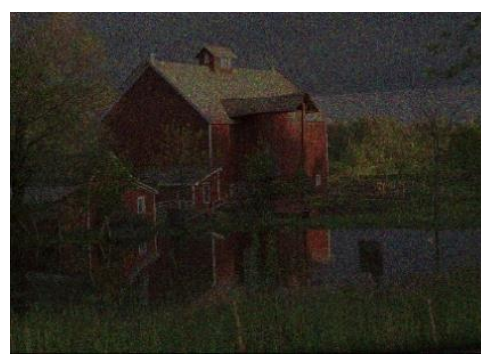

Image 11

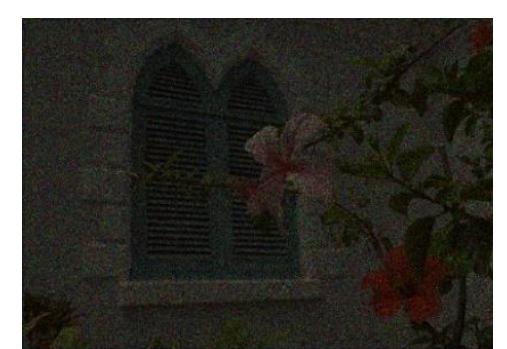

Image 3

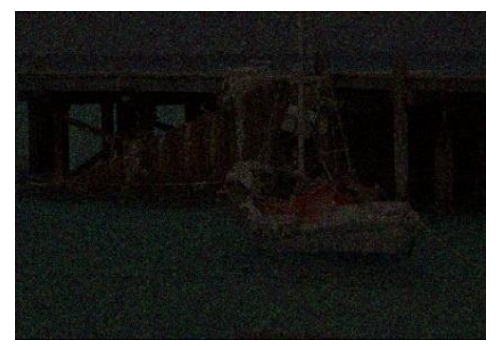

Image 6

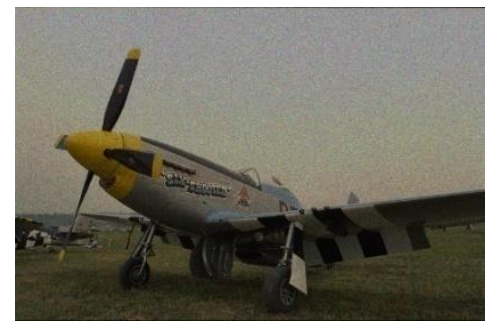

Image 9

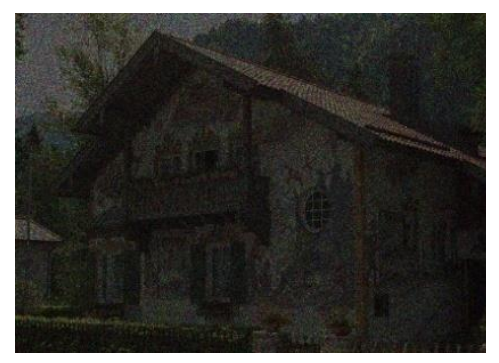

Image 12

Figure 5. Twelve noisy images at $10 \mathrm{~dB}$ from the Kodak dataset. 
Signal \& Image Processing: An International Journal (SIPIJ) Vol.11, No.5, October 2020

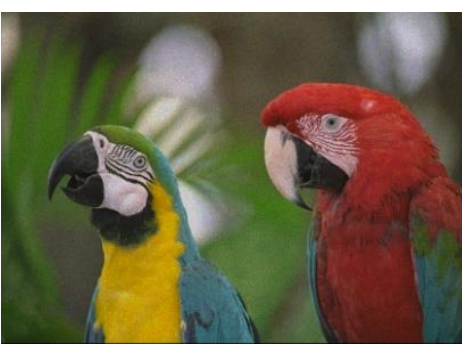

Image 1

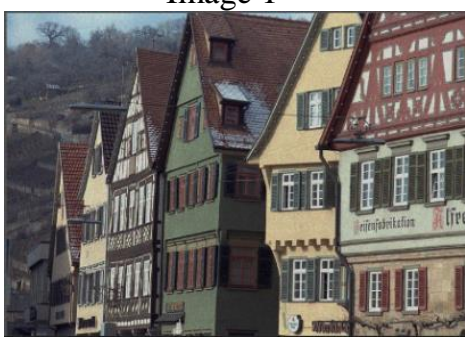

Image 4

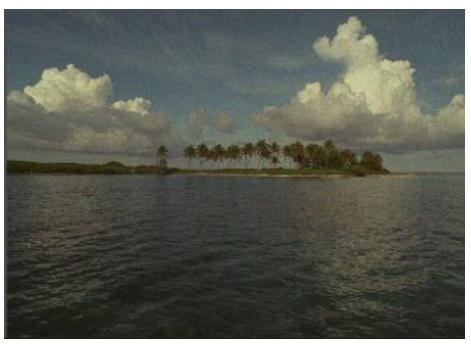

Image 7

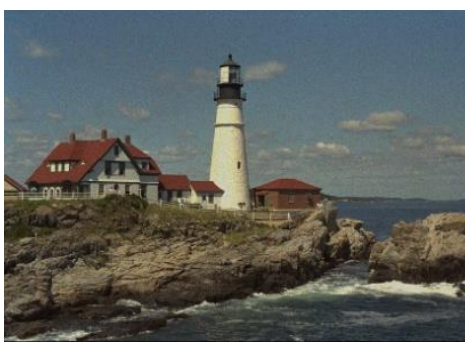

Image 10

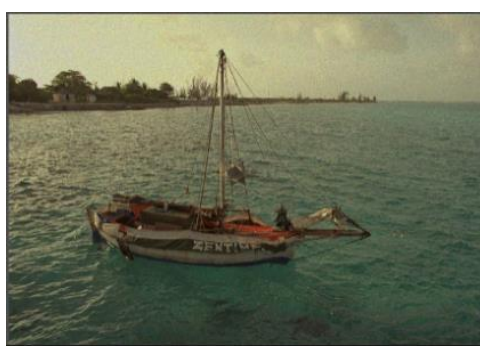

Image 2

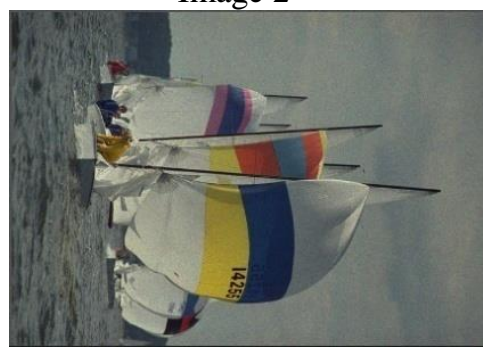

Image 5

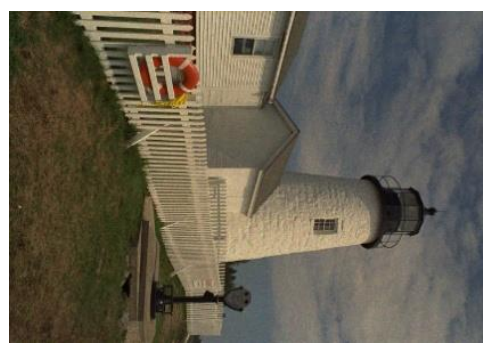

Image 8

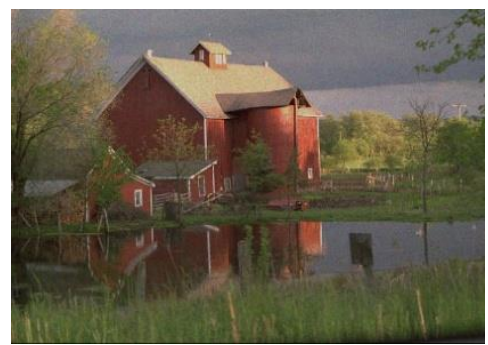

Image 11

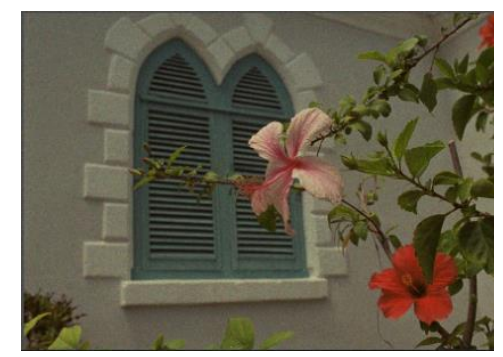

Image 3

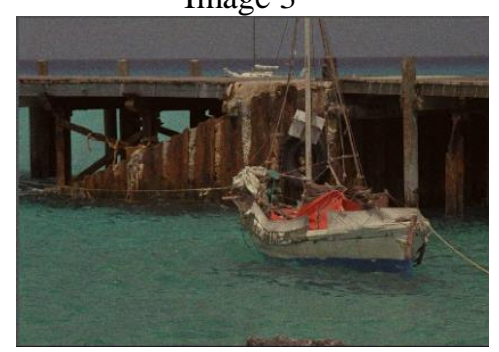

Image 6

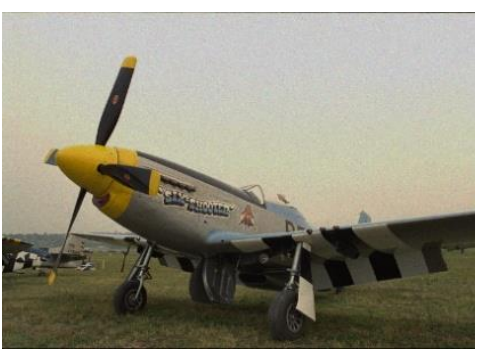

Image 9

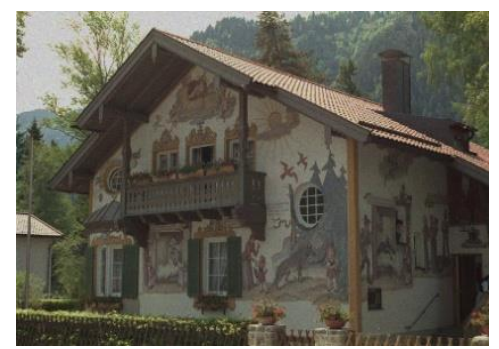

Image 12

Figure 6. Twelve noisy images at $20 \mathrm{~dB}$ from the Kodak dataset.

\subsection{Metrics}

We used the following four performance metrics to evaluate the various denoising algorithms:

- Peak Signal-to-Noise Ratio (PSNR) [39] Separate PSNRs in dBs are computed for each band. A combined PSNR is the average of the PSNRs of the individual bands. Higher PSNR values imply higher image quality.

- Human Visual System (HVS) metric Details of HVS metric in dB can be found in [40]. Higher values imply better results. 
Signal \& Image Processing: An International Journal (SIPIJ) Vol.11, No.5, October 2020

- HVSm (HVS with masking) [41] Similar to HVS, HVS incorporates the visual masking effects in computing the metrics. Higher values imply better results.

- $\quad$ IELAB

We also used CIELAB [42] for assessing demosaicing and denoising performance in our experiments. Smaller values mean good results.

It should be noted that the HVS and HVSm have better correlation with human perceptions than the other three metrics [43][44].

\section{EXPERIMENTAL RESULTS}

In our experiments, we have used the default settings in all the denoising and pansharpening algorithms.

\section{1. $10 \mathrm{~dB}$ Noisy Images}

We first present the demosaicing results without denoising in Table 1. This will form as the baseline for comparing with those denoising results later. We observe that the averaged metrics in PSNR of all methods are all around $10 \mathrm{~dB}$, meaning that demosaicing alone cannot enhance the image quality.

Table 1. Demosaicing results without denoising for $10 \mathrm{~dB}$ Poisson noisy images.

\begin{tabular}{|c|c|c|c|c|c|c|c|c|c|c|c|c|c|c|}
\hline $\begin{array}{c}\text { Ima } \\
\text { ge }\end{array}$ & & $\begin{array}{c}\text { Basel } \\
\text { ine }\end{array}$ & $\begin{array}{c}\text { Stand } \\
\text { ard }\end{array}$ & $\begin{array}{c}\text { GS } \\
\text { A }\end{array}$ & $\begin{array}{c}\text { HC } \\
\mathbf{M}\end{array}$ & $\begin{array}{c}\text { SFI } \\
\mathbf{M}\end{array}$ & $\begin{array}{c}\text { PC } \\
\text { A }\end{array}$ & $\begin{array}{c}\text { GFP } \\
\text { CA }\end{array}$ & $\begin{array}{c}\text { GL } \\
\mathbf{P}\end{array}$ & $\begin{array}{c}\mathbf{H P} \\
\mathbf{M}\end{array}$ & GS & $\begin{array}{c}\text { PRA } \\
\text { CS }\end{array}$ & $\begin{array}{c}\text { LSL } \\
\text { CD }\end{array}$ & $\begin{array}{c}\text { Bes } \\
t \\
\text { Sco } \\
\text { re }\end{array}$ \\
\hline Img & PS & & & 9.73 & 9.72 & 9.72 & 9.65 & 9.76 & 9.72 & 9.72 & 9.65 & 9.76 & 9.64 & 9.76 \\
\hline 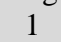 & NR & .767 & 9.730 & 6 & 1 & 2 & 5 & 1 & 8 & 1 & 3 & 4 & 3 & 7 \\
\hline & $\begin{array}{c}\text { Ciel } \\
\mathrm{ab}\end{array}$ & $\begin{array}{c}32.99 \\
3\end{array}$ & $\begin{array}{c}34.03 \\
8\end{array}$ & $\begin{array}{c}33.7 \\
19\end{array}$ & $\begin{array}{c}34.0 \\
06\end{array}$ & $\begin{array}{c}33.7 \\
09\end{array}$ & $\begin{array}{c}33.3 \\
35\end{array}$ & $\begin{array}{c}30.9 \\
69\end{array}$ & $\begin{array}{c}33.8 \\
52\end{array}$ & $\begin{array}{c}33.7 \\
10\end{array}$ & $\begin{array}{c}33.3 \\
52\end{array}$ & $\begin{array}{c}33.1 \\
67\end{array}$ & $\begin{array}{c}35.9 \\
27\end{array}$ & $\begin{array}{c}30.9 \\
69\end{array}$ \\
\hline & $\begin{array}{c}\mathrm{HV} \\
\mathrm{S}\end{array}$ & 4.162 & 4.151 & $\begin{array}{c}4.15 \\
2\end{array}$ & $\begin{array}{c}4.14 \\
2\end{array}$ & $\begin{array}{c}4.15 \\
1\end{array}$ & $\begin{array}{c}4.07 \\
0\end{array}$ & $\begin{array}{c}4.17 \\
2\end{array}$ & $\begin{array}{c}4.14 \\
6\end{array}$ & $\begin{array}{c}4.14 \\
7\end{array}$ & $\begin{array}{c}4.08 \\
2\end{array}$ & $\begin{array}{c}4.16 \\
2\end{array}$ & $\begin{array}{c}3.89 \\
1\end{array}$ & $\begin{array}{c}4.17 \\
2\end{array}$ \\
\hline & $\begin{array}{l}\mathrm{HV} \\
\mathrm{Sm}\end{array}$ & 184 & $4.1^{\prime}$ & $\begin{array}{c}4.18 \\
0\end{array}$ & $\begin{array}{c}4.17 \\
3\end{array}$ & $\begin{array}{c}4.18 \\
2\end{array}$ & $\begin{array}{c}4.09 \\
5\end{array}$ & $\begin{array}{c}4.18 \\
9\end{array}$ & $\begin{array}{c}4.17 \\
6\end{array}$ & $\begin{array}{c}4.17 \\
9\end{array}$ & $\begin{array}{c}4.10 \\
7\end{array}$ & $\begin{array}{c}4.18 \\
6\end{array}$ & $\begin{array}{c}3.91 \\
4\end{array}$ & $\begin{array}{c}4.18 \\
9\end{array}$ \\
\hline $\begin{array}{c}\operatorname{Img} \\
2\end{array}$ & $\begin{array}{l}\text { PS } \\
\text { NR }\end{array}$ & $\begin{array}{c}10.28 \\
3\end{array}$ & $\begin{array}{c}10.29 \\
4\end{array}$ & $\begin{array}{c}10.2 \\
94\end{array}$ & $\begin{array}{c}10.2 \\
90\end{array}$ & $\begin{array}{c}10.2 \\
99\end{array}$ & $\begin{array}{c}10.2 \\
23\end{array}$ & $\begin{array}{c}10.2 \\
98\end{array}$ & $\begin{array}{c}10.2 \\
95\end{array}$ & $\begin{array}{c}10.3 \\
01\end{array}$ & $\begin{array}{c}10.2 \\
28\end{array}$ & $\begin{array}{c}10.3 \\
01\end{array}$ & $\begin{array}{c}10.3 \\
21\end{array}$ & $\begin{array}{c}10.3 \\
21\end{array}$ \\
\hline & $\begin{array}{c}\text { Ciel } \\
\mathrm{ab}\end{array}$ & $\begin{array}{c}23.24 \\
5\end{array}$ & $\begin{array}{c}23.55 \\
8\end{array}$ & $\begin{array}{c}23.5 \\
22\end{array}$ & $\begin{array}{c}23.6 \\
44\end{array}$ & $\begin{array}{c}23.4 \\
78\end{array}$ & $\begin{array}{c}23.2 \\
93\end{array}$ & $\begin{array}{c}22.0 \\
80\end{array}$ & $\begin{array}{c}23.5 \\
71\end{array}$ & $\begin{array}{c}23.4 \\
79\end{array}$ & $\begin{array}{c}23.2 \\
62\end{array}$ & $\begin{array}{r}23 \\
3\end{array}$ & $\begin{array}{c}24.2 \\
07\end{array}$ & $\begin{array}{c}22.0 \\
80\end{array}$ \\
\hline & $\begin{array}{c}\mathrm{HV} \\
\mathrm{S}\end{array}$ & & & $\begin{array}{c}5.59 \\
7\end{array}$ & $\begin{array}{c}5.59 \\
7\end{array}$ & $\begin{array}{c}5.60 \\
5\end{array}$ & $\begin{array}{c}5.51 \\
6\end{array}$ & $\begin{array}{c}5.58 \\
7\end{array}$ & $\begin{array}{c}5.59 \\
8\end{array}$ & $\begin{array}{c}5.60 \\
4\end{array}$ & $\begin{array}{c}5.51 \\
8\end{array}$ & $\begin{array}{r}5.5 \\
9\end{array}$ & $\begin{array}{c}5.45 \\
8\end{array}$ & $\begin{array}{c}5.60 \\
5\end{array}$ \\
\hline & $\begin{array}{l}\mathrm{HV} \\
\mathrm{Sm}\end{array}$ & 631 & 5.644 & $\begin{array}{c}5.64 \\
4\end{array}$ & $\begin{array}{c}5.64 \\
3\end{array}$ & $\begin{array}{c}5.65 \\
2\end{array}$ & $\begin{array}{c}5.56 \\
1\end{array}$ & $\begin{array}{c}5.63 \\
1\end{array}$ & $\begin{array}{c}5.64 \\
6\end{array}$ & $\begin{array}{c}5.65 \\
2\end{array}$ & $\begin{array}{c}5.56 \\
4\end{array}$ & $\begin{array}{c}5.64 \\
5\end{array}$ & $\begin{array}{c}5.50 \\
3\end{array}$ & $\begin{array}{c}5.65 \\
2\end{array}$ \\
\hline $\begin{array}{c}\operatorname{Img} \\
3\end{array}$ & $\begin{array}{l}\text { PS } \\
\text { NR }\end{array}$ & $\begin{array}{c}0.11 \\
7\end{array}$ & $\begin{array}{c}10.06 \\
7\end{array}$ & $\begin{array}{c}10.0 \\
77\end{array}$ & $\begin{array}{c}10.0 \\
55\end{array}$ & $\begin{array}{c}10.0 \\
77\end{array}$ & $\begin{array}{c}10.0 \\
04\end{array}$ & $\begin{array}{c}10.1 \\
12\end{array}$ & $\begin{array}{c}10.0 \\
78\end{array}$ & $\begin{array}{c}10.0 \\
76\end{array}$ & $\begin{array}{c}10.0 \\
04\end{array}$ & $\begin{array}{c}10.1 \\
10\end{array}$ & $\begin{array}{c}10.0 \\
68\end{array}$ & $\begin{array}{c}10.1 \\
17\end{array}$ \\
\hline & $\begin{array}{c}\text { Ciel } \\
\mathrm{ab}\end{array}$ & $\begin{array}{c}33.36 \\
9\end{array}$ & $\begin{array}{c}35.03 \\
9\end{array}$ & $\begin{array}{c}34.3 \\
25\end{array}$ & $\begin{array}{c}34.9 \\
02\end{array}$ & $\begin{array}{c}34.0 \\
12\end{array}$ & $\begin{array}{c}33.4 \\
81\end{array}$ & $\begin{array}{c}30.0 \\
59\end{array}$ & $\begin{array}{c}34.2 \\
97\end{array}$ & $\begin{array}{c}34.0 \\
13\end{array}$ & $\begin{array}{c}33.4 \\
94\end{array}$ & $\begin{array}{c}33.6 \\
88\end{array}$ & $\begin{array}{c}33.7 \\
36\end{array}$ & $\begin{array}{c}30.0 \\
59\end{array}$ \\
\hline & $\begin{array}{c}\mathrm{HV} \\
\mathrm{S}\end{array}$ & & 370 & $\begin{array}{c}4.87 \\
5\end{array}$ & $\begin{array}{c}4.86 \\
0\end{array}$ & $\begin{array}{c}4.88 \\
1\end{array}$ & $\begin{array}{c}4.80 \\
2\end{array}$ & $\begin{array}{c}4.90 \\
9\end{array}$ & $\begin{array}{c}4.87 \\
2\end{array}$ & $\begin{array}{c}4.87 \\
7\end{array}$ & $\begin{array}{c}4.80 \\
2\end{array}$ & $\begin{array}{c}4.88 \\
5\end{array}$ & $\begin{array}{c}4.76 \\
5\end{array}$ & $\begin{array}{c}4.90 \\
9\end{array}$ \\
\hline & $\begin{array}{l}\mathrm{HV} \\
\mathrm{Sm}\end{array}$ & 922 & 4.915 & $\begin{array}{c}4.92 \\
0\end{array}$ & $\begin{array}{c}4.90 \\
9\end{array}$ & $\begin{array}{c}4.92 \\
9\end{array}$ & $\begin{array}{c}4.84 \\
2\end{array}$ & $\begin{array}{c}4.94 \\
0\end{array}$ & $\begin{array}{c}4.91 \\
9\end{array}$ & $\begin{array}{c}4.92 \\
5\end{array}$ & $\begin{array}{c}4.84 \\
3\end{array}$ & $\begin{array}{c}4.92 \\
5\end{array}$ & $\begin{array}{c}4.80 \\
4\end{array}$ & $\begin{array}{c}4.94 \\
0\end{array}$ \\
\hline $\begin{array}{c}\text { Img } \\
4\end{array}$ & $\begin{array}{l}\text { PS } \\
\text { NR }\end{array}$ & $\begin{array}{c}10.02 \\
4\end{array}$ & $\begin{array}{c}10.13 \\
1\end{array}$ & $\begin{array}{c}10.1 \\
30\end{array}$ & $\begin{array}{c}10.1 \\
26\end{array}$ & $\begin{array}{c}10.1 \\
49\end{array}$ & $\begin{array}{c}10.0 \\
18\end{array}$ & $\begin{array}{c}10.0 \\
15\end{array}$ & $\begin{array}{c}10.1 \\
42\end{array}$ & $\begin{array}{c}10.1 \\
55\end{array}$ & $\begin{array}{c}10.0 \\
20\end{array}$ & $\begin{array}{c}10.1 \\
20\end{array}$ & $\begin{array}{c}10.0 \\
57\end{array}$ & $\begin{array}{c}10.1 \\
55\end{array}$ \\
\hline & $\begin{array}{c}\text { Ciel } \\
\mathrm{ab}\end{array}$ & $\begin{array}{c}23.49 \\
9\end{array}$ & $\begin{array}{c}24.04 \\
2\end{array}$ & $\begin{array}{c}24.0 \\
37\end{array}$ & $\begin{array}{c}23.9 \\
40\end{array}$ & $\begin{array}{c}23.7 \\
97\end{array}$ & $\begin{array}{c}23.4 \\
75\end{array}$ & $\begin{array}{c}21.9 \\
25\end{array}$ & $\begin{array}{c}24.1 \\
45\end{array}$ & $\begin{array}{c}23.7 \\
97\end{array}$ & $\begin{array}{c}23.4 \\
78\end{array}$ & $\begin{array}{c}23.7 \\
70\end{array}$ & $\begin{array}{c}23.5 \\
31\end{array}$ & $\begin{array}{c}21.9 \\
25\end{array}$ \\
\hline & $\begin{array}{c}\mathrm{HV} \\
\mathrm{S}\end{array}$ & 401 & .475 & $\begin{array}{c}5.47 \\
6\end{array}$ & $\begin{array}{c}5.47 \\
5\end{array}$ & $\begin{array}{c}5.50 \\
7\end{array}$ & $\begin{array}{c}5.35 \\
1\end{array}$ & $\begin{array}{c}5.34 \\
0\end{array}$ & $\begin{array}{c}5.49 \\
9\end{array}$ & $\begin{array}{c}5.51 \\
0\end{array}$ & $\begin{array}{c}5.35 \\
2\end{array}$ & $\begin{array}{c}5.46 \\
6\end{array}$ & $\begin{array}{c}5.30 \\
2\end{array}$ & $\begin{array}{c}5.51 \\
0\end{array}$ \\
\hline
\end{tabular}


Signal \& Image Processing: An International Journal (SIPIJ) Vol.11, No.5, October 2020

\begin{tabular}{|c|c|c|c|c|c|c|c|c|c|c|c|c|c|c|}
\hline & $\begin{array}{l}\mathrm{HV} \\
\mathrm{Sm}\end{array}$ & 5.524 & 5.583 & $\begin{array}{c}5.58 \\
5\end{array}$ & $\begin{array}{c}5.58 \\
4\end{array}$ & $\begin{array}{c}5.61 \\
7\end{array}$ & $\begin{array}{c}5.46 \\
0\end{array}$ & $\begin{array}{c}5.45 \\
5\end{array}$ & $\begin{array}{c}5.60 \\
8\end{array}$ & $\begin{array}{c}5.62 \\
0\end{array}$ & $\begin{array}{c}5.46 \\
2\end{array}$ & $\begin{array}{c}5.57 \\
7\end{array}$ & $\begin{array}{c}5.41 \\
2\end{array}$ & $\begin{array}{c}5.62 \\
0\end{array}$ \\
\hline Img & PS & 10.19 & 10.15 & 10.1 & 10.1 & 10.1 & 10.0 & 10.1 & 10.1 & 10.1 & 10.0 & 10.1 & 10.1 & 10.1 \\
\hline \multirow[t]{4}{*}{5} & NR & 0 & 7 & 57 & 53 & 64 & 93 & 94 & 58 & 64 & 94 & 79 & 19 & 94 \\
\hline & $\begin{array}{c}\text { Ciel } \\
\mathrm{ab}\end{array}$ & $\begin{array}{l}4.32 \\
3\end{array}$ & $\begin{array}{l}4.67 \\
8\end{array}$ & $\begin{array}{c}24.6 \\
86\end{array}$ & $\begin{array}{c}24.6 \\
97\end{array}$ & $\begin{array}{c}24.5 \\
72\end{array}$ & $\begin{array}{c}24.4 \\
73\end{array}$ & $\begin{array}{c}23.1 \\
79\end{array}$ & $\begin{array}{c}24.6 \\
99\end{array}$ & $\begin{array}{c}24.5 \\
74\end{array}$ & $\begin{array}{c}24.4 \\
84\end{array}$ & $\begin{array}{c}24.5 \\
28\end{array}$ & $\begin{array}{c}24.8 \\
24\end{array}$ & $\begin{array}{c}23.1 \\
79\end{array}$ \\
\hline & $\begin{array}{c}\mathrm{HV} \\
\mathrm{S}\end{array}$ & & 5.946 & $\begin{array}{c}5.94 \\
5\end{array}$ & $\begin{array}{c}5.94 \\
5\end{array}$ & $\begin{array}{c}5.95 \\
4\end{array}$ & $\begin{array}{c}5.85 \\
7\end{array}$ & $\begin{array}{c}5.96 \\
6\end{array}$ & $\begin{array}{c}5.94 \\
5\end{array}$ & $\begin{array}{c}5.95 \\
1\end{array}$ & $\begin{array}{c}5.86 \\
1\end{array}$ & $\begin{array}{c}5.95 \\
4\end{array}$ & $\begin{array}{c}5.95 \\
4\end{array}$ & $\begin{array}{c}5.96 \\
6\end{array}$ \\
\hline & $\begin{array}{l}\mathrm{HV} \\
\mathrm{Sm}\end{array}$ & .989 & 5.988 & $\begin{array}{c}5.98 \\
8\end{array}$ & $\begin{array}{c}5.98 \\
7\end{array}$ & $\begin{array}{c}5.99 \\
7\end{array}$ & $\begin{array}{c}5.89 \\
6\end{array}$ & $\begin{array}{c}5.99 \\
7\end{array}$ & $\begin{array}{c}5.98 \\
9\end{array}$ & $\begin{array}{c}5.99 \\
5\end{array}$ & $\begin{array}{c}5.90 \\
1\end{array}$ & $\begin{array}{c}5.99 \\
3 \\
\end{array}$ & $\begin{array}{c}5.99 \\
4 \\
\end{array}$ & $\begin{array}{c}5.99 \\
7\end{array}$ \\
\hline \multirow[t]{4}{*}{$\begin{array}{c}\operatorname{Img} \\
6 \\
\end{array}$} & $\begin{array}{l}\text { PS } \\
\text { NR } \\
\end{array}$ & $\begin{array}{c}10.14 \\
3 \\
\end{array}$ & $\begin{array}{c}10.09 \\
8 \\
\end{array}$ & $\begin{array}{c}10.1 \\
82\end{array}$ & $\begin{array}{c}10.0 \\
60\end{array}$ & $\begin{array}{c}10.0 \\
90\end{array}$ & $\begin{array}{c}10.0 \\
55\end{array}$ & $\begin{array}{c}10.1 \\
85\end{array}$ & $\begin{array}{c}10.1 \\
66\end{array}$ & $\begin{array}{c}10.0 \\
84\end{array}$ & $\begin{array}{c}10.0 \\
56\end{array}$ & $\begin{array}{c}10.1 \\
50\end{array}$ & $\begin{array}{c}10.1 \\
70\end{array}$ & $\begin{array}{c}10.1 \\
85\end{array}$ \\
\hline & $\begin{array}{l}\text { Ciel } \\
\mathrm{ab}\end{array}$ & $\begin{array}{c}43.31 \\
2 \\
\end{array}$ & $\begin{array}{c}48.49 \\
8 \\
\end{array}$ & $\begin{array}{c}43.8 \\
84\end{array}$ & $\begin{array}{c}46.8 \\
64\end{array}$ & $\begin{array}{c}45.6 \\
45\end{array}$ & $\begin{array}{c}42.4 \\
64\end{array}$ & $\begin{array}{c}35.4 \\
42\end{array}$ & $\begin{array}{c}43.7 \\
67\end{array}$ & $\begin{array}{c}45.7 \\
30\end{array}$ & $\begin{array}{c}42.4 \\
91\end{array}$ & $\begin{array}{c}43.7 \\
65 \\
\end{array}$ & $\begin{array}{c}39.5 \\
39 \\
\end{array}$ & $\begin{array}{c}35.4 \\
42\end{array}$ \\
\hline & $\begin{array}{c}\mathrm{HV} \\
\mathrm{S} \\
\end{array}$ & 739 & 5.719 & $\begin{array}{c}5.79 \\
0 \\
\end{array}$ & $\begin{array}{c}5.70 \\
0 \\
\end{array}$ & $\begin{array}{c}5.74 \\
3 \\
\end{array}$ & $\begin{array}{c}5.68 \\
1 \\
\end{array}$ & $\begin{array}{c}5.81 \\
8 \\
\end{array}$ & $\begin{array}{c}5.78 \\
6 \\
\end{array}$ & $\begin{array}{c}5.73 \\
4 \\
\end{array}$ & $\begin{array}{c}5.68 \\
0 \\
\end{array}$ & $\begin{array}{c}5.74 \\
7 \\
\end{array}$ & $\begin{array}{c}5.78 \\
7 \\
\end{array}$ & $\begin{array}{c}5.81 \\
8 \\
\end{array}$ \\
\hline & $\begin{array}{l}\mathrm{HV} \\
\mathrm{Sm}\end{array}$ & 802 & 5.790 & $\begin{array}{c}5.86 \\
1 \\
\end{array}$ & $\begin{array}{c}5.77 \\
8 \\
\end{array}$ & $\begin{array}{c}5.82 \\
6 \\
\end{array}$ & $\begin{array}{c}5.74 \\
5 \\
\end{array}$ & $\begin{array}{c}5.86 \\
8 \\
\end{array}$ & $\begin{array}{c}5.85 \\
9 \\
\end{array}$ & $\begin{array}{c}5.81 \\
8 \\
\end{array}$ & $\begin{array}{c}5.74 \\
3 \\
\end{array}$ & $\begin{array}{c}5.81 \\
1 \\
\end{array}$ & $\begin{array}{c}5.84 \\
6 \\
\end{array}$ & $\begin{array}{c}5.86 \\
8 \\
\end{array}$ \\
\hline \multirow[t]{4}{*}{$\begin{array}{c}\text { Img } \\
7 \\
\end{array}$} & $\begin{array}{l}\text { PS } \\
\text { NR }\end{array}$ & $\begin{array}{c}0.02 \\
0 \\
\end{array}$ & 9.976 & $\begin{array}{c}9.98 \\
6 \\
\end{array}$ & $\begin{array}{c}9.96 \\
4 \\
\end{array}$ & $\begin{array}{c}9.98 \\
3 \\
\end{array}$ & $\begin{array}{c}9.89 \\
3 \\
\end{array}$ & $\begin{array}{c}10.0 \\
58 \\
\end{array}$ & $\begin{array}{c}9.98 \\
1 \\
\end{array}$ & $\begin{array}{c}9.98 \\
1 \\
\end{array}$ & $\begin{array}{c}9.89 \\
3 \\
\end{array}$ & $\begin{array}{c}10.0 \\
14 \\
\end{array}$ & $\begin{array}{c}9.99 \\
2 \\
\end{array}$ & $\begin{array}{c}10.0 \\
58 \\
\end{array}$ \\
\hline & $\begin{array}{c}\text { Ciel } \\
\mathrm{ab}\end{array}$ & $\begin{array}{c}32.93 \\
3 \\
\end{array}$ & $\begin{array}{c}34.44 \\
8 \\
\end{array}$ & $\begin{array}{c}33.9 \\
76 \\
\end{array}$ & $\begin{array}{c}34.2 \\
61 \\
\end{array}$ & $\begin{array}{c}33.5 \\
76 \\
\end{array}$ & $\begin{array}{c}33.2 \\
43 \\
\end{array}$ & $\begin{array}{c}29.2 \\
06 \\
\end{array}$ & $\begin{array}{c}33.8 \\
89 \\
\end{array}$ & $\begin{array}{c}33.5 \\
82 \\
\end{array}$ & $\begin{array}{c}33.2 \\
55\end{array}$ & $\begin{array}{c}33.3 \\
12 \\
\end{array}$ & $\begin{array}{c}32.1 \\
82 \\
\end{array}$ & $\begin{array}{c}29.2 \\
06 \\
\end{array}$ \\
\hline & $\begin{array}{c}\mathrm{HV} \\
\mathrm{S}\end{array}$ & 666 & 5.649 & $\begin{array}{c}5.65 \\
9 \\
\end{array}$ & $\begin{array}{c}5.64 \\
3 \\
\end{array}$ & $\begin{array}{c}5.65 \\
7 \\
\end{array}$ & $\begin{array}{c}5.55 \\
5\end{array}$ & $\begin{array}{c}5.72 \\
8 \\
\end{array}$ & $\begin{array}{c}5.64 \\
9 \\
\end{array}$ & $\begin{array}{c}5.65 \\
2 \\
\end{array}$ & $\begin{array}{c}5.55 \\
6 \\
\end{array}$ & $\begin{array}{c}5.66 \\
7 \\
\end{array}$ & $\begin{array}{c}5.69 \\
1 \\
\end{array}$ & $\begin{array}{c}5.72 \\
8 \\
\end{array}$ \\
\hline & $\begin{array}{l}\mathrm{HV} \\
\mathrm{Sm}\end{array}$ & 698 & 5.690 & $\begin{array}{c}5.70 \\
0 \\
\end{array}$ & $\begin{array}{c}5.68 \\
6 \\
\end{array}$ & $\begin{array}{c}5.70 \\
1 \\
\end{array}$ & $\begin{array}{c}5.59 \\
1 \\
\end{array}$ & $\begin{array}{c}5.75 \\
2 \\
\end{array}$ & $\begin{array}{c}5.69 \\
1 \\
\end{array}$ & $\begin{array}{c}5.69 \\
6 \\
\end{array}$ & $\begin{array}{c}5.59 \\
2 \\
\end{array}$ & $\begin{array}{c}5.70 \\
2 \\
\end{array}$ & $\begin{array}{c}5.72 \\
5 \\
\end{array}$ & $\begin{array}{c}5.75 \\
2 \\
\end{array}$ \\
\hline \multirow[t]{4}{*}{$\begin{array}{c}\text { Img } \\
8\end{array}$} & $\begin{array}{l}\text { PS } \\
\text { NR }\end{array}$ & .996 & 9.987 & $\begin{array}{c}9.99 \\
0\end{array}$ & $\begin{array}{c}9.98 \\
0\end{array}$ & $\begin{array}{c}9.99 \\
8\end{array}$ & $\begin{array}{c}9.90 \\
2\end{array}$ & $\begin{array}{c}10.0 \\
05\end{array}$ & $\begin{array}{c}9.99 \\
1\end{array}$ & $\begin{array}{c}9.99 \\
8\end{array}$ & $\begin{array}{c}9.90 \\
3\end{array}$ & $\begin{array}{c}10.0 \\
04\end{array}$ & $\begin{array}{c}9.96 \\
7 \\
\end{array}$ & $\begin{array}{c}10.0 \\
05\end{array}$ \\
\hline & $\begin{array}{c}\text { Ciel } \\
\mathrm{ab}\end{array}$ & $\begin{array}{c}28.77 \\
7 \\
\end{array}$ & $\begin{array}{c}29.59 \\
7 \\
\end{array}$ & $\begin{array}{c}29.3 \\
34 \\
\end{array}$ & $\begin{array}{c}29.5 \\
78 \\
\end{array}$ & $\begin{array}{c}29.1 \\
34 \\
\end{array}$ & $\begin{array}{c}28.8 \\
93 \\
\end{array}$ & $\begin{array}{c}26.5 \\
91 \\
\end{array}$ & $\begin{array}{c}29.3 \\
64 \\
\end{array}$ & $\begin{array}{c}29.1 \\
36 \\
\end{array}$ & $\begin{array}{c}28.8 \\
71 \\
\end{array}$ & $\begin{array}{c}29.0 \\
52 \\
\end{array}$ & $\begin{array}{c}29.2 \\
19 \\
\end{array}$ & $\begin{array}{c}26.5 \\
91 \\
\end{array}$ \\
\hline & $\begin{array}{c}\mathrm{HV} \\
\mathrm{S} \\
\end{array}$ & 00 & 9 & $\begin{array}{c}5.01 \\
0 \\
\end{array}$ & $\begin{array}{c}5.00 \\
7 \\
\end{array}$ & $\begin{array}{c}5.02 \\
3 \\
\end{array}$ & $\begin{array}{c}4.91 \\
1 \\
\end{array}$ & $\begin{array}{c}5.00 \\
9 \\
\end{array}$ & $\begin{array}{c}5.01 \\
1 \\
\end{array}$ & $\begin{array}{c}5.02 \\
1 \\
\end{array}$ & $\begin{array}{c}4.91 \\
3 \\
\end{array}$ & $\begin{array}{c}5.01 \\
3 \\
\end{array}$ & $\begin{array}{c}4.87 \\
7 \\
\end{array}$ & $\begin{array}{c}5.02 \\
3 \\
\end{array}$ \\
\hline & & 51 & 64 & $\begin{array}{c}5.06 \\
5\end{array}$ & $\begin{array}{c}5.06 \\
3 \\
\end{array}$ & $\begin{array}{r}5.0 \\
9 \\
\end{array}$ & $\begin{array}{c}4.96 \\
4\end{array}$ & $\begin{array}{c}5.05 \\
6\end{array}$ & $\begin{array}{c}5.0 \\
7 \\
\end{array}$ & $\begin{array}{c}5.07 \\
7 \\
\end{array}$ & $\begin{array}{c}4.96 \\
6\end{array}$ & $\begin{array}{c}5.00 \\
6 \\
\end{array}$ & $\begin{array}{c}4.92 \\
8 \\
\end{array}$ & $\begin{array}{c}5.07 \\
9 \\
\end{array}$ \\
\hline \multirow[t]{4}{*}{$\begin{array}{c}\text { Img } \\
9 \\
\end{array}$} & $\begin{array}{l}\text { PS } \\
\text { NR }\end{array}$ & $\begin{array}{c}10.09 \\
7 \\
\end{array}$ & $\begin{array}{c}10.09 \\
5 \\
\end{array}$ & $\begin{array}{c}10.0 \\
95\end{array}$ & $\begin{array}{c}10.0 \\
92\end{array}$ & 91 & 50 & $\begin{array}{c}10.1 \\
09\end{array}$ & 97 & $\begin{array}{c}10.0 \\
94\end{array}$ & $\begin{array}{c}10.0 \\
50\end{array}$ & 98 & $\begin{array}{c}10.0 \\
62 \\
\end{array}$ & $\begin{array}{c}10.1 \\
09\end{array}$ \\
\hline & $\begin{array}{c}\text { Ciel } \\
\mathrm{ab}\end{array}$ & $\begin{array}{c}16.98 \\
4 \\
\end{array}$ & $\begin{array}{c}17.07 \\
6 \\
\end{array}$ & $\begin{array}{c}17.0 \\
41 \\
\end{array}$ & $\begin{array}{c}17.1 \\
04 \\
\end{array}$ & $\begin{array}{c}17.4 \\
11 \\
\end{array}$ & $\begin{array}{c}16.8 \\
78 \\
\end{array}$ & $\begin{array}{c}16.4 \\
55 \\
\end{array}$ & $\begin{array}{c}17.1 \\
18 \\
\end{array}$ & $\begin{array}{c}17.4 \\
12 \\
\end{array}$ & $\begin{array}{c}16.8 \\
72 \\
\end{array}$ & $\begin{array}{c}17.0 \\
09 \\
\end{array}$ & $\begin{array}{c}18.1 \\
42 \\
\end{array}$ & $\begin{array}{c}16.4 \\
55 \\
\end{array}$ \\
\hline & $\begin{array}{c}\mathrm{HV} \\
\mathrm{S}\end{array}$ & 78 & 5.581 & $\begin{array}{c}5.58 \\
2 \\
\end{array}$ & $\begin{array}{c}5.58 \\
0\end{array}$ & $\begin{array}{c}5.58 \\
8 \\
\end{array}$ & $\begin{array}{c}5.54 \\
1 \\
\end{array}$ & $\begin{array}{c}5.58 \\
6\end{array}$ & $\begin{array}{c}5.58 \\
5\end{array}$ & $\begin{array}{c}5.58 \\
9 \\
\end{array}$ & $\begin{array}{c}5.54 \\
0 \\
\end{array}$ & $\begin{array}{c}5.58 \\
2 \\
\end{array}$ & $\begin{array}{c}5.32 \\
7 \\
\end{array}$ & $\begin{array}{c}5.58 \\
9 \\
\end{array}$ \\
\hline & $\begin{array}{l}\mathrm{HV} \\
\mathrm{Sm}\end{array}$ & 5.605 & 5.608 & $\begin{array}{c}5.60 \\
8\end{array}$ & $\begin{array}{c}5.60 \\
8\end{array}$ & $\begin{array}{c}5.61 \\
6\end{array}$ & $\begin{array}{c}5.56 \\
8\end{array}$ & $\begin{array}{c}5.60 \\
9\end{array}$ & $\begin{array}{c}5.61 \\
3\end{array}$ & $\begin{array}{c}5.61 \\
6\end{array}$ & $\begin{array}{c}5.56 \\
7 \\
\end{array}$ & $\begin{array}{c}5.60 \\
9\end{array}$ & $\begin{array}{c}5.35 \\
5 \\
\end{array}$ & $\begin{array}{c}5.61 \\
6\end{array}$ \\
\hline \multirow[t]{4}{*}{$\begin{array}{c}\text { Img } \\
10 \\
\end{array}$} & $\begin{array}{l}\text { PS } \\
\text { NR } \\
\end{array}$ & $\begin{array}{c}10.29 \\
4 \\
\end{array}$ & $\begin{array}{c}10.29 \\
9 \\
\end{array}$ & $\begin{array}{c}10.2 \\
99 \\
\end{array}$ & $\begin{array}{c}10.2 \\
94 \\
\end{array}$ & $\begin{array}{c}10.3 \\
06 \\
\end{array}$ & $\begin{array}{c}10.2 \\
29 \\
\end{array}$ & $\begin{array}{c}10.2 \\
93 \\
\end{array}$ & $\begin{array}{r}10.3 \\
02 \\
\end{array}$ & $\begin{array}{c}10.3 \\
08 \\
\end{array}$ & $\begin{array}{c}10.2 \\
28 \\
\end{array}$ & $\begin{array}{c}10.3 \\
07 \\
\end{array}$ & $\begin{array}{c}10.2 \\
45 \\
\end{array}$ & $\begin{array}{c}10.3 \\
08 \\
\end{array}$ \\
\hline & $\begin{array}{c}\text { Ciel } \\
\mathrm{ab}\end{array}$ & $\begin{array}{c}26.00 \\
0\end{array}$ & $\begin{array}{c}26.43 \\
1 \\
\end{array}$ & $\begin{array}{c}26.3 \\
81\end{array}$ & $\begin{array}{c}26.4 \\
35\end{array}$ & $\begin{array}{c}26.2 \\
48\end{array}$ & $\begin{array}{c}25.9 \\
78\end{array}$ & $\begin{array}{c}24.6 \\
79\end{array}$ & $\begin{array}{c}26.4 \\
48\end{array}$ & $\begin{array}{c}26.2 \\
49\end{array}$ & $\begin{array}{c}26.0 \\
24\end{array}$ & $\begin{array}{c}26.1 \\
89 \\
\end{array}$ & $\begin{array}{c}26.3 \\
15 \\
\end{array}$ & $\begin{array}{c}24.6 \\
79\end{array}$ \\
\hline & $\begin{array}{c}\mathrm{HV} \\
\mathrm{S} \\
\end{array}$ & & 15 & $\begin{array}{c}6.31 \\
3 \\
\end{array}$ & $\begin{array}{c}6.31 \\
4 \\
\end{array}$ & $\begin{array}{c}6.33 \\
1 \\
\end{array}$ & $\begin{array}{c}6.25 \\
2 \\
\end{array}$ & $\begin{array}{c}6.28 \\
7 \\
\end{array}$ & $\begin{array}{c}6.31 \\
9 \\
\end{array}$ & $\begin{array}{c}6.33 \\
1 \\
\end{array}$ & $\begin{array}{c}6.24 \\
6 \\
\end{array}$ & $\begin{array}{c}6.31 \\
5 \\
\end{array}$ & $\begin{array}{c}6.32 \\
7 \\
\end{array}$ & $\begin{array}{c}6.33 \\
1 \\
\end{array}$ \\
\hline & $\begin{array}{l}\mathrm{HV} \\
\mathrm{Sm}\end{array}$ & 362 & 6.374 & $\begin{array}{c}6.37 \\
3 \\
\end{array}$ & $\begin{array}{c}6.37 \\
4 \\
\end{array}$ & $\begin{array}{c}6.39 \\
1 \\
\end{array}$ & $\begin{array}{c}6.31 \\
2 \\
\end{array}$ & $\begin{array}{c}6.34 \\
4\end{array}$ & $\begin{array}{c}6.37 \\
9 \\
\end{array}$ & $\begin{array}{c}6.39 \\
1 \\
\end{array}$ & $\begin{array}{c}6.30 \\
6\end{array}$ & $\begin{array}{c}6.37 \\
4 \\
\end{array}$ & $\begin{array}{c}6.38 \\
9 \\
\end{array}$ & $\begin{array}{c}6.39 \\
1 \\
\end{array}$ \\
\hline \multirow[t]{4}{*}{$\begin{array}{c}\text { Img } \\
11 \\
\end{array}$} & $\begin{array}{l}\text { PS } \\
\text { NR }\end{array}$ & $\begin{array}{c}10.44 \\
2 \\
\end{array}$ & $\begin{array}{c}10.41 \\
1 \\
\end{array}$ & $\begin{array}{c}10.4 \\
14\end{array}$ & $\begin{array}{c}10.4 \\
04\end{array}$ & $\begin{array}{c}10.4 \\
17\end{array}$ & $\begin{array}{c}10.3 \\
36\end{array}$ & $\begin{array}{c}10.4 \\
48\end{array}$ & $\begin{array}{c}10.4 \\
13\end{array}$ & $\begin{array}{c}10.4 \\
17\end{array}$ & $\begin{array}{c}10.3 \\
36\end{array}$ & $\begin{array}{c}10.4 \\
38\end{array}$ & $\begin{array}{c}10.3 \\
84\end{array}$ & $\begin{array}{c}10.4 \\
48\end{array}$ \\
\hline & $\begin{array}{c}\text { Ciel } \\
\mathrm{ab}\end{array}$ & $\begin{array}{c}28.61 \\
1 \\
\end{array}$ & $\begin{array}{c}29.43 \\
1 \\
\end{array}$ & $\begin{array}{c}29.2 \\
09 \\
\end{array}$ & $\begin{array}{c}29.4 \\
41 \\
\end{array}$ & $\begin{array}{c}29.0 \\
23 \\
\end{array}$ & $\begin{array}{c}28.7 \\
87 \\
\end{array}$ & $\begin{array}{c}26.5 \\
37 \\
\end{array}$ & $\begin{array}{c}29.2 \\
57\end{array}$ & $\begin{array}{c}29.0 \\
24 \\
\end{array}$ & $\begin{array}{c}28.7 \\
75 \\
\end{array}$ & $\begin{array}{c}28.8 \\
15 \\
\end{array}$ & $\begin{array}{c}29.5 \\
63 \\
\end{array}$ & $\begin{array}{c}26.5 \\
37 \\
\end{array}$ \\
\hline & $\begin{array}{c}\mathrm{HV} \\
\mathrm{S}\end{array}$ & 251 & 5.242 & $\begin{array}{c}5.24 \\
2 \\
\end{array}$ & $\begin{array}{c}5.23 \\
6\end{array}$ & $\begin{array}{c}5.25 \\
1\end{array}$ & $\begin{array}{c}5.17 \\
3 \\
\end{array}$ & $\begin{array}{c}5.26 \\
6\end{array}$ & $\begin{array}{c}5.24 \\
0\end{array}$ & $\begin{array}{c}5.24 \\
8\end{array}$ & $\begin{array}{c}5.17 \\
3 \\
\end{array}$ & $\begin{array}{c}5.25 \\
1 \\
\end{array}$ & $\begin{array}{c}5.03 \\
2 \\
\end{array}$ & $\begin{array}{c}5.26 \\
6\end{array}$ \\
\hline & $\begin{array}{l}\mathrm{HV} \\
\mathrm{Sm}\end{array}$ & 5.285 & 5.283 & $\begin{array}{c}5.28 \\
3\end{array}$ & $\begin{array}{c}5.27 \\
9\end{array}$ & $\begin{array}{c}5.29 \\
3\end{array}$ & $\begin{array}{c}5.21 \\
1\end{array}$ & $\begin{array}{c}5.29 \\
6\end{array}$ & $\begin{array}{c}5.28 \\
1\end{array}$ & $\begin{array}{c}5.29 \\
0\end{array}$ & $\begin{array}{c}5.21 \\
1\end{array}$ & $\begin{array}{c}5.28 \\
8\end{array}$ & $\begin{array}{c}5.06 \\
7\end{array}$ & $\begin{array}{c}5.29 \\
6\end{array}$ \\
\hline
\end{tabular}


Signal \& Image Processing: An International Journal (SIPIJ) Vol.11, No.5, October 2020

\begin{tabular}{|c|c|c|c|c|c|c|c|c|c|c|c|c|c|c|}
\hline $\begin{array}{r}\mathrm{Im} \\
12 \\
\end{array}$ & $\begin{array}{l}\text { PS } \\
\text { NR } \\
\end{array}$ & $\begin{array}{c}0.14 \\
2 \\
\end{array}$ & $\begin{array}{c}0.13 \\
4 \\
\end{array}$ & $\begin{array}{c}10.1 \\
37 \\
\end{array}$ & $\begin{array}{c}10.1 \\
26 \\
\end{array}$ & $\begin{array}{c}10.1 \\
46 \\
\end{array}$ & $\begin{array}{c}10.0 \\
62 \\
\end{array}$ & $\begin{array}{c}10.1 \\
40 \\
\end{array}$ & $\begin{array}{c}10.1 \\
39 \\
\end{array}$ & $\begin{array}{c}10.1 \\
47 \\
\end{array}$ & $\begin{array}{c}10.0 \\
62 \\
\end{array}$ & $\begin{array}{c}10.1 \\
50 \\
\end{array}$ & $\begin{array}{c}10.1 \\
25 \\
\end{array}$ & $\begin{array}{c}10.1 \\
50 \\
\end{array}$ \\
\hline & $\begin{array}{c}\text { Ciel } \\
\mathrm{ab}\end{array}$ & $\begin{array}{c}29.85 \\
1\end{array}$ & $\begin{array}{c}30.86 \\
4 \\
\end{array}$ & $\begin{array}{c}30.6 \\
46\end{array}$ & $\begin{array}{c}30.7 \\
99\end{array}$ & $\begin{array}{c}30.3 \\
07\end{array}$ & $\begin{array}{c}29.7 \\
91\end{array}$ & $\begin{array}{c}27.0 \\
68\end{array}$ & $\begin{array}{c}30.6 \\
45\end{array}$ & $\begin{array}{c}30.3 \\
11 \\
\end{array}$ & $\begin{array}{c}29.8 \\
01 \\
\end{array}$ & $\begin{array}{c}30.2 \\
04\end{array}$ & $\begin{array}{c}29.6 \\
12\end{array}$ & $\begin{array}{c}27.0 \\
68 \\
\end{array}$ \\
\hline & $\begin{array}{c}\mathrm{HV} \\
\mathrm{S}\end{array}$ & 447 & 5.446 & $\begin{array}{c}5.44 \\
8 \\
\end{array}$ & $\begin{array}{c}5.44 \\
2 \\
\end{array}$ & $\begin{array}{c}5.46 \\
2 \\
\end{array}$ & $\begin{array}{c}5.37 \\
3 \\
\end{array}$ & $\begin{array}{c}5.45 \\
0 \\
\end{array}$ & $\begin{array}{c}5.44 \\
9 \\
\end{array}$ & $\begin{array}{c}5.46 \\
0 \\
\end{array}$ & $\begin{array}{c}5.37 \\
4 \\
\end{array}$ & $\begin{array}{c}5.45 \\
3 \\
\end{array}$ & $\begin{array}{c}5.39 \\
1 \\
\end{array}$ & $\begin{array}{c}5.46 \\
2 \\
\end{array}$ \\
\hline & $\begin{array}{l}\mathrm{HV} \\
\mathrm{Sm} \\
\end{array}$ & 5.504 & 5.506 & $\begin{array}{c}5.50 \\
7 \\
\end{array}$ & $\begin{array}{c}5.50 \\
3 \\
\end{array}$ & $\begin{array}{c}5.52 \\
2 \\
\end{array}$ & $\begin{array}{c}5.43 \\
1 \\
\end{array}$ & $\begin{array}{c}5.50 \\
3 \\
\end{array}$ & $\begin{array}{c}5.50 \\
9 \\
\end{array}$ & $\begin{array}{c}5.52 \\
0 \\
\end{array}$ & $\begin{array}{c}5.43 \\
2 \\
\end{array}$ & $\begin{array}{c}5.51 \\
0 \\
\end{array}$ & $\begin{array}{c}5.44 \\
7 \\
\end{array}$ & $\begin{array}{c}5.52 \\
2 \\
\end{array}$ \\
\hline $\begin{array}{c}\text { Ave } \\
- \\
\text { rage }\end{array}$ & $\begin{array}{l}\text { PS } \\
\text { NR } \\
\end{array}$ & $\begin{array}{c}10.12 \\
6\end{array}$ & $\begin{array}{c}10.11 \\
5 \\
\end{array}$ & $\begin{array}{c}10.1 \\
25 \\
\end{array}$ & $\begin{array}{c}10.1 \\
05 \\
\end{array}$ & $\begin{array}{c}10.1 \\
20 \\
\end{array}$ & $\begin{array}{c}10.0 \\
43 \\
\end{array}$ & $\begin{array}{c}10.1 \\
35 \\
\end{array}$ & $\begin{array}{c}10.1 \\
24 \\
\end{array}$ & $\begin{array}{c}10.1 \\
20 \\
\end{array}$ & $\begin{array}{c}10.0 \\
44 \\
\end{array}$ & $\begin{array}{r}10 \\
3 \\
\end{array}$ & $\begin{array}{c}10.0 \\
96 \\
\end{array}$ & $\begin{array}{c}10.1 \\
36 \\
\end{array}$ \\
\hline & $\begin{array}{c}\text { Ciel } \\
\mathrm{ab}\end{array}$ & $\begin{array}{c}28.65 \\
8 \\
\end{array}$ & $\begin{array}{c}29.80 \\
8 \\
\end{array}$ & $\begin{array}{c}29.2 \\
30\end{array}$ & $\begin{array}{c}29.6 \\
39 \\
\end{array}$ & $\begin{array}{c}29.2 \\
43 \\
\end{array}$ & $\begin{array}{c}28.6 \\
74\end{array}$ & $\begin{array}{c}26.1 \\
82\end{array}$ & $\begin{array}{c}29.2 \\
54\end{array}$ & $\begin{array}{c}29.2 \\
51\end{array}$ & $\begin{array}{c}28.6 \\
80\end{array}$ & $\begin{array}{c}28.9 \\
11\end{array}$ & $\begin{array}{c}28.9 \\
00\end{array}$ & $\begin{array}{c}26.1 \\
82 \\
\end{array}$ \\
\hline & $\begin{array}{c}\mathrm{HV} \\
\mathrm{S}\end{array}$ & 5.414 & 5.417 & $\begin{array}{c}5.42 \\
4 \\
\end{array}$ & $\begin{array}{c}5.41 \\
2 \\
\end{array}$ & $\begin{array}{c}5.43 \\
0 \\
\end{array}$ & $\begin{array}{c}5.34 \\
0 \\
\end{array}$ & $\begin{array}{c}5.42 \\
6 \\
\end{array}$ & $\begin{array}{c}5.42 \\
5 \\
\end{array}$ & $\begin{array}{c}5.42 \\
7 \\
\end{array}$ & $\begin{array}{c}5.34 \\
1 \\
\end{array}$ & $\begin{array}{c}5.42 \\
4\end{array}$ & $\begin{array}{c}5.31 \\
7\end{array}$ & $\begin{array}{c}5.43 \\
0\end{array}$ \\
\hline & $\begin{array}{l}\mathrm{HV} \\
\mathrm{Sm} \\
\end{array}$ & 5.463 & 5.469 & $\begin{array}{c}5.47 \\
6 \\
\end{array}$ & $\begin{array}{c}5.46 \\
6 \\
\end{array}$ & $\begin{array}{c}5.48 \\
4 \\
\end{array}$ & $\begin{array}{c}5.39 \\
0 \\
\end{array}$ & $\begin{array}{c}5.47 \\
0 \\
\end{array}$ & $\begin{array}{c}5.47 \\
8 \\
\end{array}$ & $\begin{array}{c}5.48 \\
1 \\
\end{array}$ & $\begin{array}{c}5.39 \\
1 \\
\end{array}$ & $\begin{array}{c}5.47 \\
4 \\
\end{array}$ & $\begin{array}{c}5.36 \\
5 \\
\end{array}$ & $\begin{array}{c}5.48 \\
4 \\
\end{array}$ \\
\hline
\end{tabular}

For the results obtained from different denoising filters, instead of showing big tables like Table 1 above, we extracted the best performing results from those big tables and create summarized tables. Table 2 summarizes the best BM3D filtering results for three denoising configurations. It can be seen that the combination of GFPCA and post-denoising has the best performance. The PSNR value has been improved from $10 \mathrm{~dB}$ to $17.9 \mathrm{~dB}$.

Table 3 summarizes the best wavelet denoising results for three denoising configurations. We can see that hybrid denoising has slight edge over the other configurations. The PSNR value has been improved from $10 \mathrm{~dB}$ to $17 \mathrm{~dB}$. Table 4 summarizes the best diffusion denoising results for the three denoising configurations. It can be seen that the results are worse than other denoising algorithms. Table 5 to Table 7 summarize the median filtering results. We can observe that the $7 \times 7$ option achieved the best among the three median filters. Actually, the best performing method is the hybrid denoising using $7 \times 7$ median filter with GFPCA and the PSNR value has reached $22 \mathrm{~dB}$ from $10 \mathrm{~dB}$. This is quite remarkable. Table 8 summarizes the FFDNET results. The performance is better than BM3D, wavelet, and diffusion, but worse than those median filters.

We also include some denoised images for the pre-denoising case in Figure 7. The post-denoising and hybrid denoising results can be found in Fig. A1 and Fig. A2 of the Appendix. It can be seen that the median filter with $7 \times 7$ size has the closest intensity to the ground truth. BM3D, wavelet, and FFDNET all have smooth results, but somehow their images look darker than the ground truth.

Table 2. Best performing BM3D denoising results for $10 \mathrm{~dB}$ noisy images. Bold numbers indicate the best in each row.

\begin{tabular}{|c|c|c|c|}
\hline Metrics & $\begin{array}{c}\text { Hybrid Denoising/ } \\
\text { Best Algorithm }\end{array}$ & $\begin{array}{c}\text { Post-Denoising / Best } \\
\text { Algorithm }\end{array}$ & $\begin{array}{c}\text { Pre-Denoising / Best } \\
\text { Algorithm }\end{array}$ \\
\hline PSNR (dB) & $17.565 /$ GFPCA & $\mathbf{1 7 . 9 0 1 / G F P C A ~}$ & $15.768 /$ GFPCA \\
\hline CIELAB & $10.414 /$ GFPCA & $\mathbf{1 0 . 2 0 9 / G F P C A ~}$ & $12.975 /$ GFPCA \\
\hline HVS $(\mathrm{dB})$ & $12.847 /$ GFPCA & $\mathbf{1 3 . 2 2 8 / G F P C A ~}$ & 11.058 /GFPCA \\
\hline HVSm $(\mathrm{dB})$ & $13.038 /$ GFPCA & $\mathbf{1 3 . 4 3 6 / G F P C A ~}$ & $11.203 /$ GFPCA \\
\hline
\end{tabular}


Signal \& Image Processing: An International Journal (SIPIJ) Vol.11, No.5, October 2020

Table 3. Best performing wavelet denoising results for $10 \mathrm{~dB}$ noisy images. Bold numbers indicate the best in each row.

\begin{tabular}{|c|c|c|c|}
\hline Metrics & $\begin{array}{c}\text { Hybrid Denoising/ } \\
\text { Best Algorithm }\end{array}$ & $\begin{array}{c}\text { Post-Denoising / } \\
\text { Best Algorithm }\end{array}$ & $\begin{array}{c}\text { Pre-Denoising / } \\
\text { Best Algorithm }\end{array}$ \\
\hline PSNR $(\mathrm{dB})$ & $\mathbf{1 7 . 0 1 2 / B a s e l i n e}$ & $15.331 /$ Standard & $16.612 / \mathrm{GFPCA}$ \\
\hline CIELAB & $11.997 /$ GFPCA & $\mathbf{1 2 . 8 6 0 / G F P C A}$ & $11.887 / \mathrm{GFPCA}$ \\
\hline HVS $(\mathrm{dB})$ & $\mathbf{1 1 . 9 5 5 / B a s e l i n e}$ & $10.511 /$ GFPCA & $11.599 / \mathrm{GFPCA}$ \\
\hline HVSm $(\mathrm{dB})$ & $\mathbf{1 2 . 1 7 7 / B a s e l i n e}$ & $10.641 / \mathrm{GFPCA}$ & $11.775 / \mathrm{GFPCA}$ \\
\hline
\end{tabular}

Table 4: Best performing diffusion filter denoising results for $10 \mathrm{~dB}$ noisy images. Bold numbers indicate the best in each row.

\begin{tabular}{|c|c|c|c|}
\hline Metrics & $\begin{array}{c}\text { Hybrid Denoising/ } \\
\text { Best Algorithm }\end{array}$ & $\begin{array}{c}\text { Post-Denoising / } \\
\text { Best Algorithm }\end{array}$ & $\begin{array}{c}\text { Pre-Denoising / } \\
\text { Best Algorithm }\end{array}$ \\
\hline PSNR (dB) & $\mathbf{1 6 . 3 9 3 / B a s e l i n e ~}$ & $15.353 /$ Standard & $14.822 /$ GFPCA \\
\hline CIELAB & $13.374 /$ GFPCA & $13.353 /$ GFPCA & $\mathbf{1 4 . 4 9 0 / G F P C A ~}$ \\
\hline HVS $(\mathrm{dB})$ & $\mathbf{1 1 . 3 1 8 / B a s e l i n e ~}$ & $10.466 /$ Standard & $9.851 /$ GFPCA \\
\hline HVSm $(\mathrm{dB})$ & $\mathbf{1 1 . 5 2 4 / B a s e l i n e}$ & $10.652 /$ Standard & $9.969 /$ GFPCA \\
\hline
\end{tabular}

Table 5. Best performing median filter (3x3) denoising results for $10 \mathrm{~dB}$ noisy images. Bold numbers indicate the best in each row.

\begin{tabular}{|c|c|c|c|}
\hline Metrics & $\begin{array}{c}\text { Hybrid Denoising/ } \\
\text { Best Algorithm }\end{array}$ & $\begin{array}{c}\text { Post-Denoising / } \\
\text { Best Algorithm }\end{array}$ & $\begin{array}{c}\text { Pre-Denoising / } \\
\text { Best Algorithm }\end{array}$ \\
\hline PSNR (dB) & $19.362 /$ GFPCA & $\mathbf{1 9 . 4 6 7 / G F P C A ~}$ & $18.841 /$ GFPCA \\
\hline CIELAB & $8.905 /$ GFPCA & $\mathbf{8 . 4 7 5 / G F P C A ~}$ & $9.438 /$ GFPCA \\
\hline HVS (dB) & $14.444 /$ GFPCA & $\mathbf{1 4 . 8 0 4 / G F P C A ~}$ & $13.963 /$ GFPCA \\
\hline HVSm $(\mathrm{dB})$ & $14.777 / G F P C A$ & $\mathbf{1 5 . 1 3 8 / G F P C A ~}$ & $14.288 /$ GFPCA \\
\hline
\end{tabular}

Table 6. Best performing median filter $(5 \times 5)$ denoising results for $10 \mathrm{~dB}$ noisy images. Bold numbers indicate the best in each row.

\begin{tabular}{|c|c|c|c|}
\hline Metrics & $\begin{array}{c}\text { Hybrid Denoising/ } \\
\text { Best Algorithm }\end{array}$ & $\begin{array}{c}\text { Post-Denoising / } \\
\text { Best Algorithm }\end{array}$ & $\begin{array}{c}\text { Pre-Denoising / } \\
\text { Best Algorithm }\end{array}$ \\
\hline PSNR (dB) & $\mathbf{2 1 . 6 4 7 / G F P C A ~}$ & $21.218 /$ GFPCA & $21.405 / G F P C A$ \\
\hline CIELAB & $\mathbf{7 . 3 1 2 / G F P C A ~}$ & $7.376 /$ GFPCA & $7.550 /$ GFPCA \\
\hline HVS (dB) & $\mathbf{1 6 . 7 9 1 / G F P C A ~}$ & $16.531 /$ GFPCA & $16.632 / G F P C A$ \\
\hline HVSm (dB) & $\mathbf{1 7 . 3 9 9 / G F P C A ~}$ & $17.069 / G F P C A$ & $17.266 / G F P C A$ \\
\hline
\end{tabular}

Table 7. Best performing median filter (7x7) denoising results for $10 \mathrm{~dB}$ noisy images. Bold numbers indicate the best in each row.

\begin{tabular}{|c|c|c|c|}
\hline Metrics & $\begin{array}{c}\text { Hybrid Denoising/ } \\
\text { Best Algorithm }\end{array}$ & $\begin{array}{c}\text { Post-Denoising / } \\
\text { Best Algorithm }\end{array}$ & $\begin{array}{c}\text { Pre-Denoising / } \\
\text { Best Algorithm }\end{array}$ \\
\hline PSNR (dB) & $\mathbf{2 2 . 1 0 2 / G F P C A ~}$ & $21.552 /$ GFPCA & $21.927 /$ GFPCA \\
\hline CIELAB & $\mathbf{7 . 0 3 5 / G F P C A ~}$ & $7.140 /$ GFPCA & $7.257 /$ GFPCA \\
\hline HVS (dB) & $\mathbf{1 7 . 1 9 4 / G F P C A ~}$ & $16.708 /$ GFPCA & $17.073 /$ GFPCA \\
\hline HVSm $(\mathrm{dB})$ & $\mathbf{1 7 . 8 5 7 / G F P C A ~}$ & $17.295 /$ GFPCA & $17.757 /$ GFPCA \\
\hline
\end{tabular}


Signal \& Image Processing: An International Journal (SIPIJ) Vol.11, No.5, October 2020

Table 8. Best performing FFDNET denoising results for $10 \mathrm{~dB}$ noisy images. Bold numbers indicate the best in each row.

\begin{tabular}{|c|c|c|c|}
\hline Metrics & $\begin{array}{c}\text { Hybrid Denoising/ } \\
\text { Best Algorithm }\end{array}$ & $\begin{array}{c}\text { Post-Denoising / } \\
\text { Best Algorithm }\end{array}$ & $\begin{array}{c}\text { Pre-Denoising / } \\
\text { Best Algorithm }\end{array}$ \\
\hline PSNR (dB) & $17.761 /$ GFPCA & $\mathbf{1 8 . 1 3 1 / G F P C A ~}$ & $17.020 / \mathrm{HPM}$ \\
\hline CIELAB & $10.686 /$ GFPCA & $\mathbf{9 . 8 9 6 / G F P C A ~}$ & $11.655 / \mathrm{GFPCA}$ \\
\hline HVS $(\mathrm{dB})$ & $13.123 / \mathrm{GFPCA}$ & $\mathbf{1 3 . 5 7 2 / G F P C A}$ & $12.309 / \mathrm{HPM}$ \\
\hline HVSm $(\mathrm{dB})$ & $13.342 / \mathrm{GFPCA}$ & $\mathbf{1 3 . 7 9 7 / G F P C A}$ & $12.506 / \mathrm{HPM}$ \\
\hline
\end{tabular}

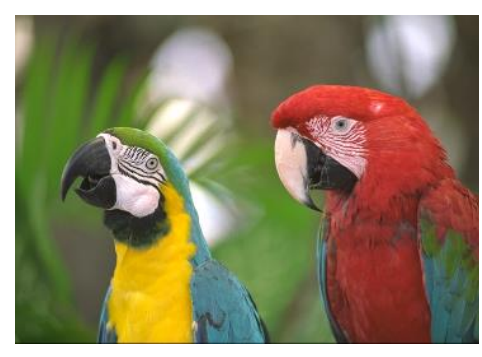

GT

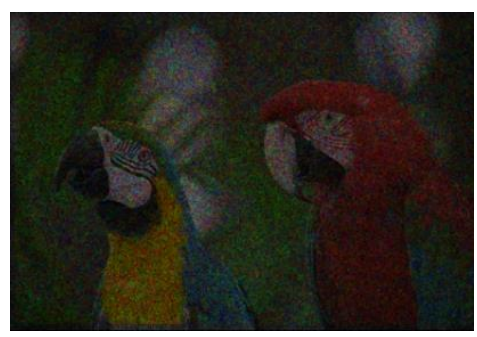

No Denoising/PRACS

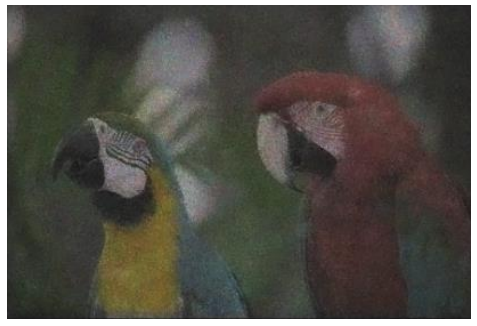

Diffusion/GFPCA

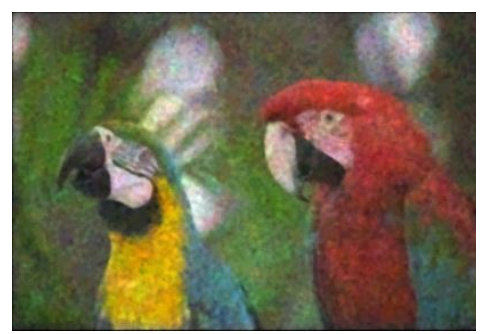

Medfilt 7x7/GFPCA

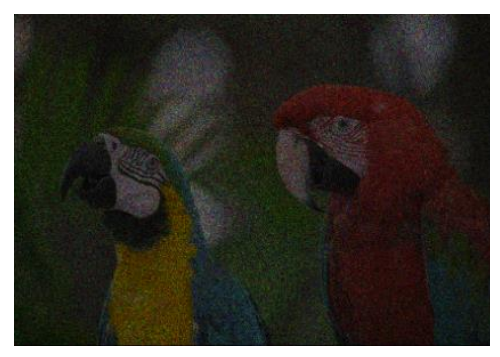

Noisy Input

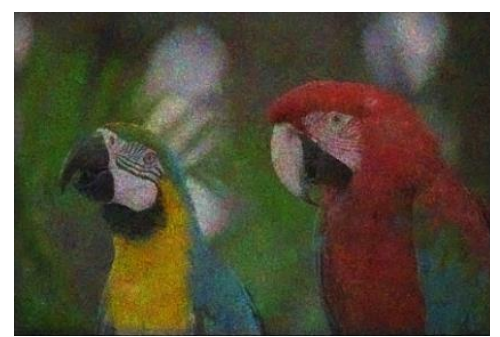

BM3D/GFPCA

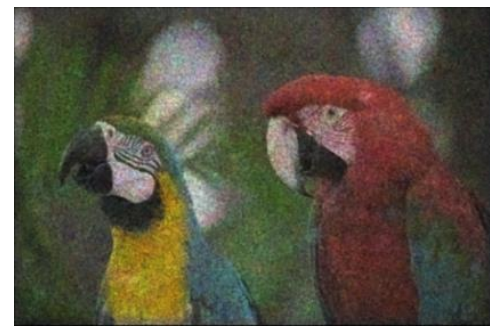

Medfilt 3x3/GFPCA

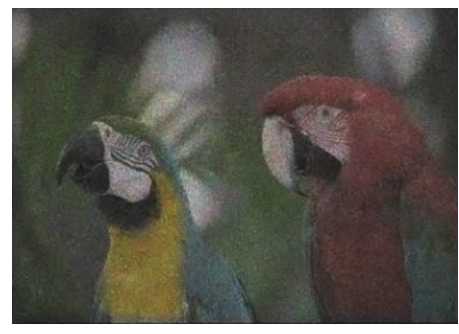

Wavelet/GFPCA

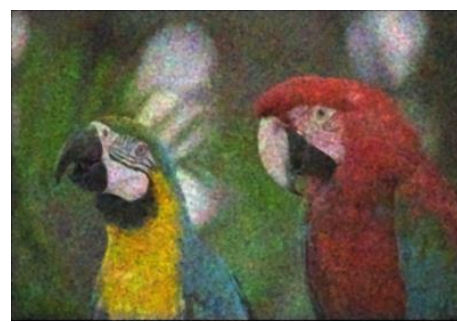

Medfilt 5x5/GFPCA

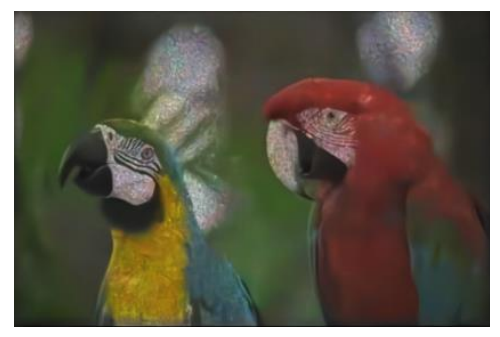

FFDNET/HPM

Figure 7. Demosaicing results using various pre-denoising approaches for $10 \mathrm{~dB}$ noisy images. For each image, $a / b$ means the "a" is the denoising method and " $b$ " is the pansharpening method. 
Signal \& Image Processing: An International Journal (SIPIJ) Vol.11, No.5, October 2020

\section{2. $20 \mathrm{~dB}$ Noisy Images}

We first present the demosaicing results without denoising in Table 9. This will help the comparison among those denoising results later. We observe that the averaged metrics in PSNR of all methods are all less than $20 \mathrm{~dB}$, meaning that demosaicing alone cannot enhance the image quality.

Table 9. Demosaicing results without denoising for $20 \mathrm{~dB}$ Poisson noisy images.

\begin{tabular}{|c|c|c|c|c|c|c|c|c|c|c|c|c|c|c|}
\hline Image & & Baseline & Standard & GSA & HCM & SFIM & PCA & GFPCA & GLP & HPM & GS & PRACS & LSLCD & $\begin{array}{c}\text { Best } \\
\text { Score } \\
\end{array}$ \\
\hline \multirow[t]{4}{*}{ Img1 } & PSNR & 19.977 & 20.037 & 20.038 & 20.011 & 19.934 & 19.933 & 19.856 & 20.023 & 19.943 & 19.932 & 20.050 & 19.557 & 20.050 \\
\hline & Cielab & 7.721 & 7.767 & 7.771 & 7.841 & 8.106 & 7.713 & 7.976 & 7.854 & 8.108 & 7.745 & 7.706 & 9.384 & 7.706 \\
\hline & HVS & 14.529 & 14.558 & 14.558 & 14.541 & 14.547 & 14.384 & 14.376 & 14.557 & 14.548 & 14.470 & 14.562 & 13.590 & 14.562 \\
\hline & HVSm & 14.620 & 14.638 & 14.638 & 14.627 & 14.635 & 14.461 & 14.457 & 14.643 & 14.637 & 14.549 & 14.641 & 13.669 & 14.643 \\
\hline \multirow[t]{4}{*}{ Img2 } & PSNR & 18.519 & 18.973 & 18.967 & 18.954 & 18.948 & 18.791 & 18.750 & 18.955 & 18.962 & 18.814 & 18.925 & 19.088 & 19.088 \\
\hline & Cielab & 7.730 & 7.595 & 7.603 & 7.685 & 7.649 & 7.542 & 7.074 & 7.650 & 7.648 & 7.520 & 7.631 & 7.434 & 7.074 \\
\hline & HVS & 14.106 & 14.363 & 14.377 & 14.368 & 14.393 & 14.185 & 14.109 & 14.385 & 14.392 & 14.196 & 14.355 & 14.114 & 14.393 \\
\hline & HVSm & 14.334 & 14.505 & 14.513 & 14.506 & 14.531 & 14.317 & 14.298 & 14.527 & 14.533 & 14.332 & 14.503 & 14.232 & 14.533 \\
\hline \multirow[t]{4}{*}{ Img3 } & PSNR & 20.181 & 20.301 & 20.302 & 20.271 & 20.310 & 20.165 & 20.183 & 20.327 & 20.324 & 20.167 & 20.297 & 20.105 & 20.327 \\
\hline & Cielab & 7.956 & 7.968 & 7.896 & 8.119 & 8.083 & 7.849 & 7.779 & 7.980 & 8.084 & 7.854 & 7.933 & 8.441 & 7.779 \\
\hline & HVS & 15.101 & 15.176 & 15.177 & 15.175 & 15.212 & 15.041 & 15.045 & 15.211 & 15.216 & 15.050 & 15.173 & 14.713 & 15.216 \\
\hline & HVSm & 15.245 & 15.292 & 15.292 & 15.290 & 15.324 & 15.158 & 15.164 & 15.324 & 15.328 & 15.168 & 15.292 & 14.824 & 15.328 \\
\hline \multirow[t]{4}{*}{ Img4 } & PSNR & 18.011 & 19.443 & 19.438 & 19.420 & 19.420 & 18.993 & 18.654 & 19.402 & 19.451 & 19.003 & 19.312 & 19.498 & 19.498 \\
\hline & Cielab & 8.009 & 7.417 & 7.440 & 7.429 & 7.413 & 7.284 & 6.832 & 7.608 & 7.416 & 7.282 & 7.421 & 7.394 & 6.832 \\
\hline & HVS & 14.118 & 14.942 & 14.952 & 14.957 & 15.050 & 14.468 & 14.090 & 15.016 & 15.054 & 14.467 & 14.866 & 14.770 & 15.054 \\
\hline & HVSm & 14.726 & 15.290 & 15.296 & 15.299 & 15.416 & 14.823 & 14.551 & 15.379 & 15.423 & 14.825 & 15.252 & 15.081 & 15.423 \\
\hline \multirow[t]{4}{*}{ Img5 } & PSNR & 20.048 & 20.211 & 20.209 & 20.196 & 20.200 & 20.052 & 20.151 & 20.204 & 20.208 & 20.068 & 20.212 & 20.031 & 20.212 \\
\hline & Cielab & 6.604 & 6.585 & 6.566 & 6.593 & 6.591 & 6.571 & 6.348 & 6.606 & 6.592 & 6.567 & 6.569 & 6.802 & 6.348 \\
\hline & HVS & 15.873 & 16.000 & 16.001 & 15.997 & 16.019 & 15.787 & 15.866 & 16.016 & 16.018 & 15.824 & 15.996 & 15.892 & 16.019 \\
\hline & HVSm & 16.044 & 16.126 & 16.127 & 16.123 & 16.144 & 15.911 & 15.998 & 16.145 & 16.146 & 15.951 & 16.126 & 16.027 & 16.146 \\
\hline \multirow[t]{4}{*}{ Img6 } & PSNR & 20.041 & 20.433 & 20.431 & 20.402 & 20.423 & 20.237 & 20.228 & 20.433 & 20.437 & 20.240 & 20.393 & 20.362 & 20.437 \\
\hline & Cielab & 8.710 & 8.668 & 8.620 & 8.756 & 8.645 & 8.369 & 7.628 & 8.744 & 8.653 & 8.385 & 8.629 & 8.495 & 7.628 \\
\hline & HVS & 15.852 & 16.095 & 16.080 & 16.105 & 16.153 & 15.946 & 15.785 & 16.121 & 16.157 & 15.919 & 16.046 & 15.884 & 16.157 \\
\hline & HVSm & 16.159 & 16.321 & 16.311 & 16.325 & 16.377 & 16.181 & 16.030 & 16.352 & 16.382 & 16.151 & 16.293 & 16.091 & 16.382 \\
\hline \multirow[t]{4}{*}{ Img7 } & PSNR & 19.969 & 20.154 & 20.154 & 20.141 & 20.136 & 20.002 & 20.098 & 20.139 & 20.144 & 20.010 & 20.152 & 20.110 & 20.154 \\
\hline & Cielab & 7.741 & 7.691 & 7.693 & 7.752 & 7.717 & 7.677 & 6.996 & 7.724 & 7.716 & 7.666 & 7.705 & 7.341 & 6.996 \\
\hline & HVS & 15.752 & 15.874 & 15.875 & 15.875 & 15.880 & 15.711 & 15.805 & 15.866 & 15.878 & 15.720 & 15.870 & 15.852 & 15.880 \\
\hline & HVSm & 15.908 & 15.997 & 15.997 & 15.996 & 16.006 & 15.829 & 15.938 & 15.994 & 16.005 & 15.839 & 15.994 & 15.968 & 16.006 \\
\hline \multirow[t]{4}{*}{ Img8 } & $\begin{array}{l}\text { PSNR } \\
\end{array}$ & 19.518 & 20.122 & 20.120 & 20.090 & 20.103 & 19.849 & 19.767 & 20.107 & 20.110 & 19.865 & 20.039 & 20.090 & 20.122 \\
\hline & Cielab & 7.622 & 7.473 & 7.419 & 7.580 & 7.468 & 7.370 & 6.919 & 7.500 & 7.472 & 7.361 & 7.507 & 7.316 & 6.919 \\
\hline & HVS & 14.901 & 15.295 & 15.310 & 15.317 & 15.357 & 15.015 & 14.918 & 15.334 & 15.349 & 15.032 & 15.257 & 14.869 & 15.357 \\
\hline & HVSm & 15.181 & 15.473 & 15.483 & 15.486 & 15.531 & 15.189 & 15.142 & 15.514 & 15.527 & 15.210 & 15.453 & 15.030 & 15.531 \\
\hline \multirow[t]{4}{*}{ Img9 } & PSNR & 15.927 & 15.998 & 15.998 & 15.991 & 15.982 & 15.919 & 15.981 & 16.003 & 15.986 & 15.917 & 15.995 & 15.951 & 16.003 \\
\hline & Cielab & 8.286 & 8.227 & 8.187 & 8.254 & 8.587 & 8.100 & 7.971 & 8.262 & 8.607 & 8.095 & 8.190 & 8.484 & 7.971 \\
\hline & HVS & 11.458 & 11.503 & 11.502 & 11.502 & 11.519 & 11.431 & 11.469 & 11.516 & 11.522 & 11.428 & 11.498 & 11.284 & 11.522 \\
\hline & HVSm & 11.529 & 11.555 & 11.555 & 11.554 & 11.571 & 11.483 & 11.522 & 11.567 & 11.573 & 11.480 & 11.553 & 11.336 & 11.573 \\
\hline \multirow[t]{4}{*}{ Img10 } & $\begin{array}{l}\text { PSNR } \\
\end{array}$ & 19.541 & 20.006 & 20.004 & 19.985 & 20.003 & 19.793 & 19.801 & 20.006 & 20.017 & 19.789 & 19.949 & 19.968 & 20.017 \\
\hline & Cielab & 7.567 & 7.421 & 7.415 & 7.477 & 7.406 & 7.333 & 6.861 & 7.519 & 7.406 & 7.351 & 7.427 & 7.331 & 6.861 \\
\hline & HVS & 15.738 & 16.043 & 16.024 & 16.057 & 16.110 & 15.856 & 15.737 & 16.075 & 16.114 & 15.822 & 15.982 & 16.037 & 16.114 \\
\hline & HVSm & 16.060 & 16.248 & 16.238 & 16.253 & 16.309 & 16.078 & 15.984 & 16.283 & 16.314 & 16.042 & 16.217 & 16.233 & 16.314 \\
\hline \multirow[t]{4}{*}{ Img11 } & $\begin{array}{l}\text { PSNR } \\
\end{array}$ & 19.862 & 20.142 & 20.141 & 20.111 & 20.121 & 19.983 & 19.959 & 20.134 & 20.132 & 19.981 & 20.115 & 20.019 & 20.142 \\
\hline & Cielab & 7.759 & 7.710 & 7.703 & 7.815 & 7.765 & 7.660 & 7.476 & 7.760 & 7.762 & 7.656 & 7.675 & 8.033 & 7.476 \\
\hline & HVS & 14.955 & 15.074 & 15.075 & 15.070 & 15.093 & 14.940 & 14.875 & 15.086 & 15.093 & 14.936 & 15.064 & 14.610 & 15.093 \\
\hline & HVSm & 15.126 & 15.203 & 15.203 & 15.200 & 15.223 & 15.068 & 15.021 & 15.217 & 15.224 & 15.064 & 15.199 & 14.730 & 15.224 \\
\hline \multirow[t]{4}{*}{$\operatorname{Img} 12$} & PSNR & 19.405 & 20.032 & 20.031 & 19.998 & 20.017 & 19.818 & 19.720 & 20.030 & 20.039 & 19.820 & 19.970 & 20.208 & 20.208 \\
\hline & Cielab & 7.571 & 7.473 & 7.468 & 7.538 & 7.483 & 7.277 & 6.947 & 7.522 & 7.483 & 7.279 & 7.462 & 7.322 & 6.947 \\
\hline & HVS & 15.389 & 15.636 & 15.636 & 15.638 & 15.675 & 15.426 & 15.380 & 15.660 & 15.681 & 15.429 & 15.620 & 15.471 & 15.681 \\
\hline & HVSm & 15.704 & 15.844 & 15.844 & 15.843 & 15.887 & 15.634 & 15.617 & 15.873 & 15.894 & 15.637 & 15.840 & 15.639 & 15.894 \\
\hline \multirow[t]{4}{*}{$\begin{array}{l}\text { Ave- } \\
\text { rage }\end{array}$} & PSNR & 19.250 & 19.654 & 19.653 & 19.631 & 19.633 & 19.461 & 19.429 & 19.647 & 19.646 & 19.467 & 19.618 & 19.582 & 19.654 \\
\hline & Cielab & 7.773 & 7.666 & 7.649 & 7.737 & 7.743 & 7.562 & 7.234 & 7.727 & 7.746 & 7.563 & 7.655 & 7.815 & 7.234 \\
\hline & HVS & 14.814 & 15.047 & 15.047 & 15.050 & 15.084 & 14.849 & 14.788 & 15.070 & 15.085 & 14.858 & 15.024 & 14.757 & 15.085 \\
\hline & HVSm & 15.053 & 15.208 & 15.208 & 15.208 & 15.246 & 15.011 & 14.977 & 15.235 & 15.249 & 15.021 & 15.197 & 14.905 & 15.249 \\
\hline
\end{tabular}


Signal \& Image Processing: An International Journal (SIPIJ) Vol.11, No.5, October 2020

Table 10 summarizes the best BM3D filtering results for three denoising configurations. It can be seen that pre-denoising has the best performance. The PSNR value has been improved from 20 $\mathrm{dB}$ to $27.128 \mathrm{~dB}$. Table 11 summarizes the best wavelet denoising results for three denoising configurations. We can see that hybrid denoising has slight edge over the other configurations. The PSNR value has been improved from $20 \mathrm{~dB}$ to $27 \mathrm{~dB}$. Table 12 summarizes the best diffusion denoising results for the three denoising configurations. It can be seen that the results are worse than other denoising algorithms. Table 13 to Table 15 summarize the median filtering results. We can observe that the $3 \times 3$ option achieved the best among the three median filters. However, the median filter results are worse than BM3D and wavelet approaches. Table 16 summarizes the FFDNET results. The performance is better than BM3D, wavelet, and diffusion, but worse than those median filters.

We include some denoised images for the pre-denoising case in Figure 8. The post-denoising and hybrid denoising results can be found in Fig. A3 and Fig. A4 of the Appendix. It can be seen that the BM3D and medial filters have close resemblance to the ground truth. The wavelet and diffusion filter look dark as compared to the ground truth. Finally, FFDNET has over smoothed results.

Table 10. Best performing BM3D denoising results for $20 \mathrm{~dB}$ noisy images. Bold numbers indicate the best in each row.

\begin{tabular}{|c|c|c|c|}
\hline Metrics & $\begin{array}{c}\text { Hybrid Denoising/ } \\
\text { Best Algorithm }\end{array}$ & $\begin{array}{c}\text { Post-Denoising / } \\
\text { Best Algorithm }\end{array}$ & $\begin{array}{c}\text { Pre-Denoising / } \\
\text { Best Algorithm }\end{array}$ \\
\hline PSNR (dB) & $27.122 /$ Standard & $24.963 /$ GFPCA & 27.128/GSA \\
\hline CIELAB & $3.845 /$ Standard & $4.326 /$ GFPCA & $\mathbf{3 . 6 8 0 / G F P C A ~}$ \\
\hline HVS (dB) & $\mathbf{2 3 . 0 0 2 / S t a n d a r d ~}$ & $20.623 /$ GFPCA & $23.071 /$ SFIM \\
\hline HVSm (dB) & $23.895 /$ Standard & 21.394 GFPCA & $\mathbf{2 3 . 9 9 2 / S F I M ~}$ \\
\hline
\end{tabular}

Table 11. Best performing wavelet denoising results for $20 \mathrm{~dB}$ noisy images. Bold numbers indicate the best in each row.

\begin{tabular}{|c|c|c|c|}
\hline Met & $\begin{array}{l}\text { Hybrid Denoising/ } \\
\text { Best Algorithm }\end{array}$ & $\begin{array}{l}\text { Post-Denoising / } \\
\text { Best Algorithm }\end{array}$ & $\begin{array}{l}\text { Pre-Denoising / } \\
\text { Best Algorithm }\end{array}$ \\
\hline & & $23.364 / \mathrm{GFPCA}$ & 26.830/Standard \\
\hline & & A & $\overline{\mathbf{C A}}$ \\
\hline & & $\mathrm{CA}$ & $\mathrm{IM}$ \\
\hline $\operatorname{HVSm}(\mathrm{dB})$ & 23.477/SFIM & 21.394/GFPCA & 23.469/SFIM \\
\hline
\end{tabular}

Table 12. Best performing diffusion filter denoising results for $20 \mathrm{~dB}$ noisy images. Bold numbers indicate the best in each row.

\begin{tabular}{|c|c|c|c|}
\hline Metrics & $\begin{array}{c}\text { Hybrid Denoising/ } \\
\text { Best Algorithm }\end{array}$ & $\begin{array}{c}\text { Post-Denoising / } \\
\text { Best Algorithm }\end{array}$ & $\begin{array}{c}\text { Pre-Denoising / } \\
\text { Best Algorithm }\end{array}$ \\
\hline PSNR (dB) & $\mathbf{2 5 . 5 1 9 / G S A ~}$ & $23.178 /$ GFPCA & $25.367 /$ Standard \\
\hline CIELAB & $5.415 /$ GFPCA & 5.016/GFPCA & $5.242 /$ GFPCA \\
\hline HVS (dB) & $\mathbf{2 0 . 8 8 7 / G S A ~}$ & $18.614 /$ GFPCA & $20.702 /$ GSA \\
\hline HVSm (dB) & $\mathbf{2 1 . 5 1 1 / G S A ~}$ & $19.047 /$ GFPCA & $21.298 / G S A$ \\
\hline
\end{tabular}


Signal \& Image Processing: An International Journal (SIPIJ) Vol.11, No.5, October 2020

Table 13. Best performing median filter ( $3 \times 3)$ denoising results for $20 \mathrm{~dB}$ noisy images. Bold numbers indicate the best in each row.

\begin{tabular}{|c|c|c|c|}
\hline Metrics & $\begin{array}{c}\text { Hybrid Denoising/ } \\
\text { Best Algorithm }\end{array}$ & $\begin{array}{c}\text { Post-Denoising / } \\
\text { Best Algorithm }\end{array}$ & $\begin{array}{c}\text { Pre-Denoising / } \\
\text { Best Algorithm }\end{array}$ \\
\hline PSNR (dB) & $26.654 /$ Standard & $25.282 /$ GFPCA & 26.661/GSA \\
\hline CIELAB & $3.644 /$ GFPCA & $3.929 /$ GFPCA & $\mathbf{3 . 5 8 0 / G F P C A ~}$ \\
\hline HVS (dB) & $23.094 /$ HCM & $21.221 /$ GFPCA & $\mathbf{2 3 . 1 6 9 / S t a n d a r d ~}$ \\
\hline HVSm (dB) & $24.419 /$ Standard & $22.219 /$ GFPCA & $\mathbf{2 4 . 5 0 5 / S F I M ~}$ \\
\hline
\end{tabular}

Table 14. Best performing median filter $(5 \times 5)$ denoising results for $20 \mathrm{~dB}$ noisy images. Bold numbers indicate the best in each row.

\begin{tabular}{|c|c|c|c|}
\hline Metrics & $\begin{array}{c}\text { Hybrid Denoising/ } \\
\text { Best Algorithm }\end{array}$ & $\begin{array}{c}\text { Post-Denoising / } \\
\text { Best Algorithm }\end{array}$ & $\begin{array}{c}\text { Pre-Denoising / } \\
\text { Best Algorithm }\end{array}$ \\
\hline PSNR (dB) & $24.962 /$ Standard & $24.889 /$ GFPCA & $\mathbf{2 5 . 0 0 1 / G L P ~}$ \\
\hline CIELAB & $3.994 /$ GFPCA & $\mathbf{3 . 8 8 6}$ GFPCA & $3.907 /$ GFPCA \\
\hline HVS $(\mathrm{dB})$ & $21.247 /$ Standard & $20.735 /$ GFPCA & $\mathbf{2 1 . 3 7 7 / S F I M ~}$ \\
\hline HVSm $(\mathrm{dB})$ & $22.493 /$ Standard & $21.889 /$ GFPCA & $\mathbf{2 2 . 6 4 8 / S F I M ~}$ \\
\hline
\end{tabular}

Table 15. Best performing median filter (7x7) denoising results for $20 \mathrm{~dB}$ noisy images. Bold numbers indicate the best in each row.

\begin{tabular}{|c|c|c|c|}
\hline Metrics & $\begin{array}{c}\text { Hybrid Denoising/ } \\
\text { Best Algorithm }\end{array}$ & $\begin{array}{c}\text { Post-Denoising / } \\
\text { Best Algorithm }\end{array}$ & $\begin{array}{c}\text { Pre-Denoising / } \\
\text { Best Algorithm }\end{array}$ \\
\hline PSNR (dB) & $23.710 /$ Standard & $24.346 /$ Baseline & $\mathbf{2 3 . 7 6 8 / G L P ~}$ \\
\hline CIELAB & $\mathbf{4 . 4 5 3 / G F P C A ~}$ & $4.057 /$ GFPCA & $4.344 /$ GFPCA \\
\hline HVS $(\mathrm{dB})$ & $19.445 /$ Standard & $\mathbf{1 9 . 9 6 3 / B a s e l i n e ~}$ & $19.550 /$ GLP \\
\hline HVSm (dB) & $20.438 /$ Standard & $\mathbf{2 1 . 0 2 7 / B a s e l i n e ~}$ & $20.558 /$ GLP \\
\hline
\end{tabular}

Table 16. Best performing FFDNET denoising results for $20 \mathrm{~dB}$ noisy images. Bold numbers indicate the best in each row.

\begin{tabular}{|c|c|c|c|}
\hline Metrics & $\begin{array}{c}\text { Hybrid Denoising/ } \\
\text { Best Algorithm }\end{array}$ & $\begin{array}{c}\text { Post-Denoising / } \\
\text { Best Algorithm }\end{array}$ & $\begin{array}{c}\text { Pre-Denoising / } \\
\text { Best Algorithm }\end{array}$ \\
\hline PSNR (dB) & $26.674 /$ Standard & $24.686 /$ GFPCA & $\mathbf{2 6 . 6 7 6 / G S A ~}$ \\
\hline CIELAB & $3.916 /$ Standard & $4.533 /$ GFPCA & 3.914/GSA \\
\hline HVS (dB) & $22.854 /$ Standard & $20.444 /$ GFPCA & $\mathbf{2 2 . 9 6 0 / S F I M ~}$ \\
\hline HVSm (dB) & $23.994 /$ Standard & $21.161 /$ GFPCA & $\mathbf{2 4 . 1 2 4 / S F I M ~}$ \\
\hline
\end{tabular}


Signal \& Image Processing: An International Journal (SIPIJ) Vol.11, No.5, October 2020

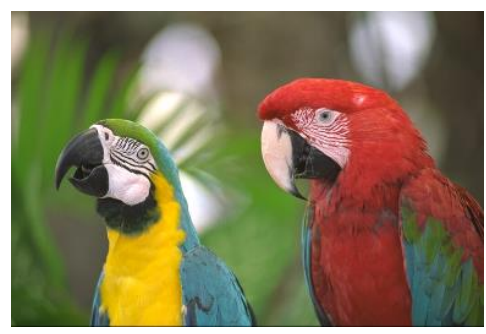

GT

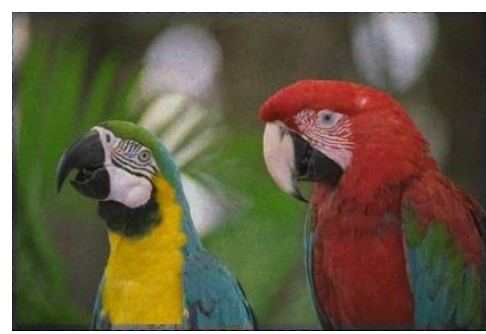

No Denoising/Standard

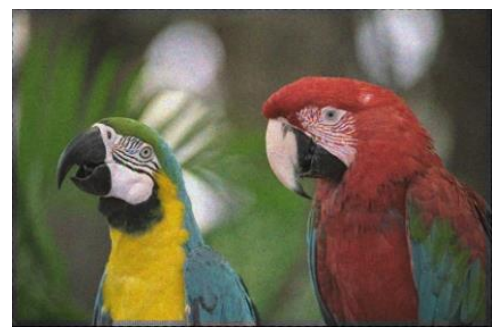

Diffusion/Standard

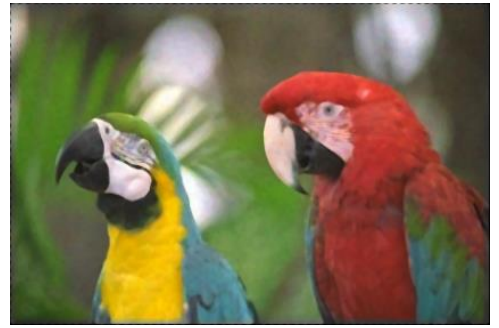

Medfilt 7x7/GLP

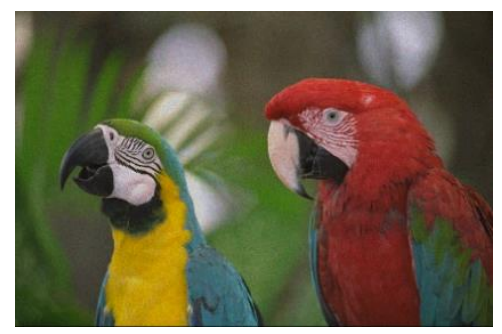

Noisy Input

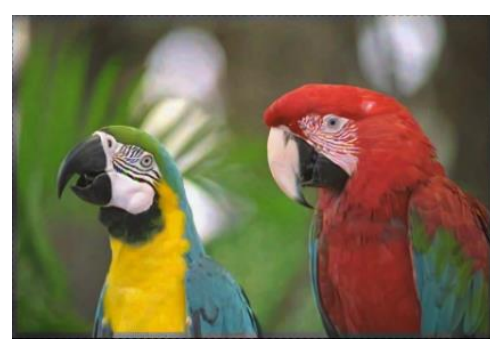

BM3D/GSA

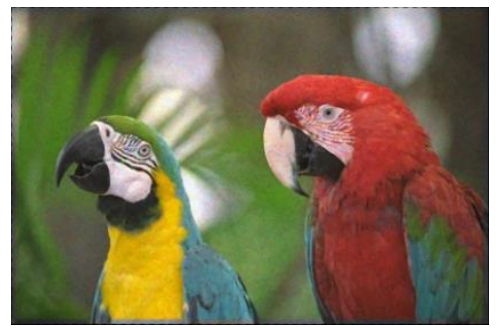

Medfilt 3x3/GSA

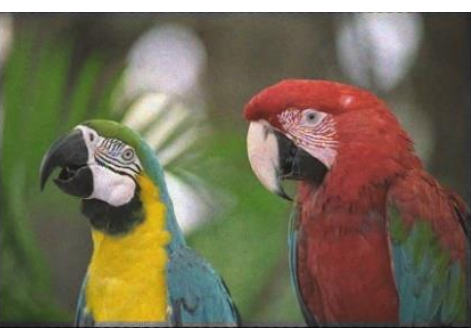

Wavelet/Standard

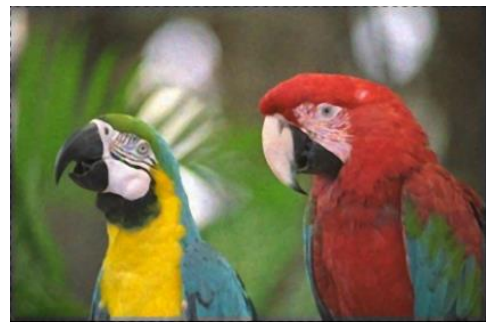

Medfilt 5x5/GLP

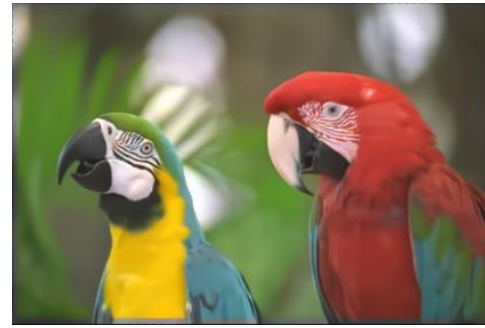

\section{FFDNET/GSA}

Figure 8. Demosaicing results using various pre-denoising approaches for $20 \mathrm{~dB}$ noisy images. For each image, a/b means the "a" is the denoising method and " $\mathrm{b}$ " is the pansharpening method.

\subsection{Discussions}

\subsection{1. $10 \mathrm{~dB}$ case}

From the results in Sections 3.1 and 3.2, we have following observations:

- All filters improved over the no filtering case.

- Median filter with 7x7 has the best performance in all four metrics. It has improved the PSNR by more than $10 \mathrm{dBs}$. 
Signal \& Image Processing: An International Journal (SIPIJ) Vol.11, No.5, October 2020

- Median filter with 5x5 is the second best.

- The worst filter is the diffusion filter.

- Pre-filtering is better than post-filtering in wavelet, and median filters with $5 \times 5$ and $7 \times 7$ sizes. However, other filters have opposite behavior.

- $\quad$ FFDNET did not yield better performance than conventional filers.

- Hybrid did not yield additional gains over either pre-filtering or post-filtering.

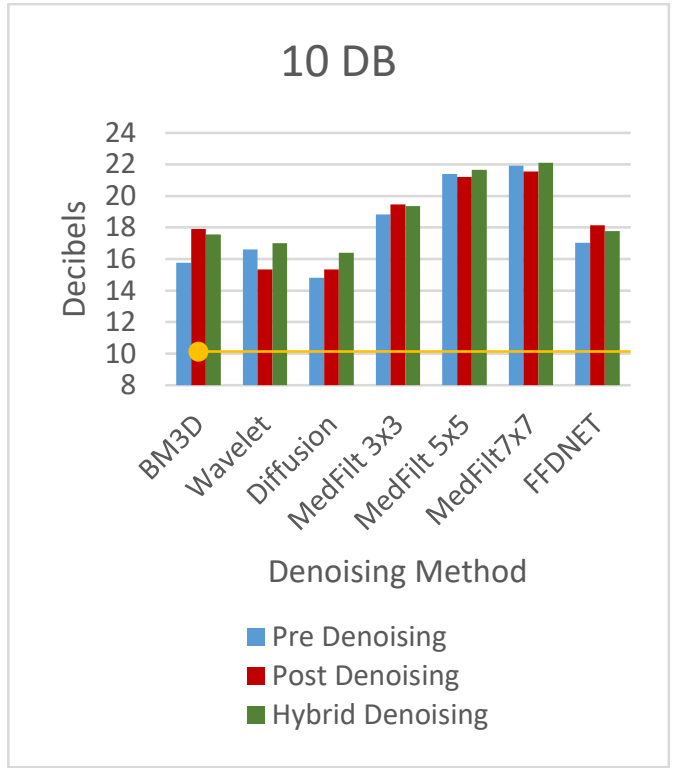

(a) PSNR

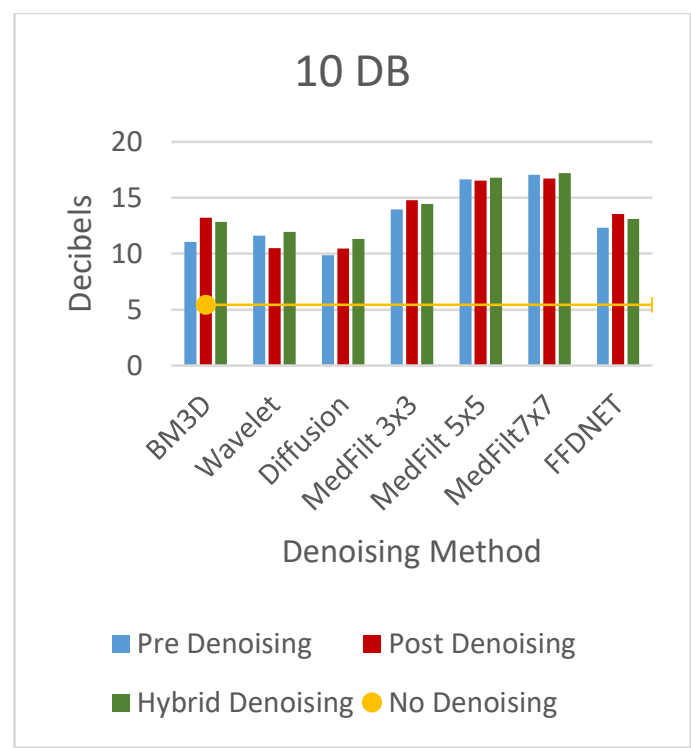

(c) HVS

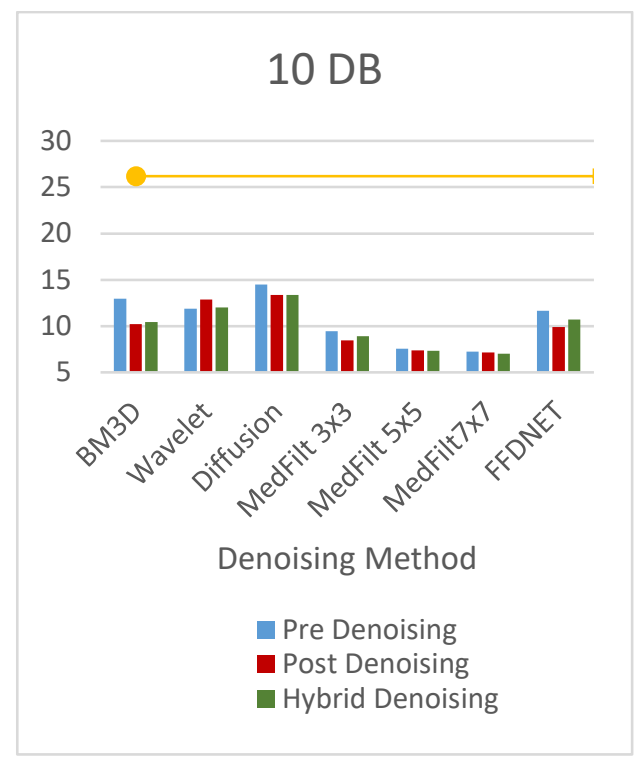

(b) CIELAB

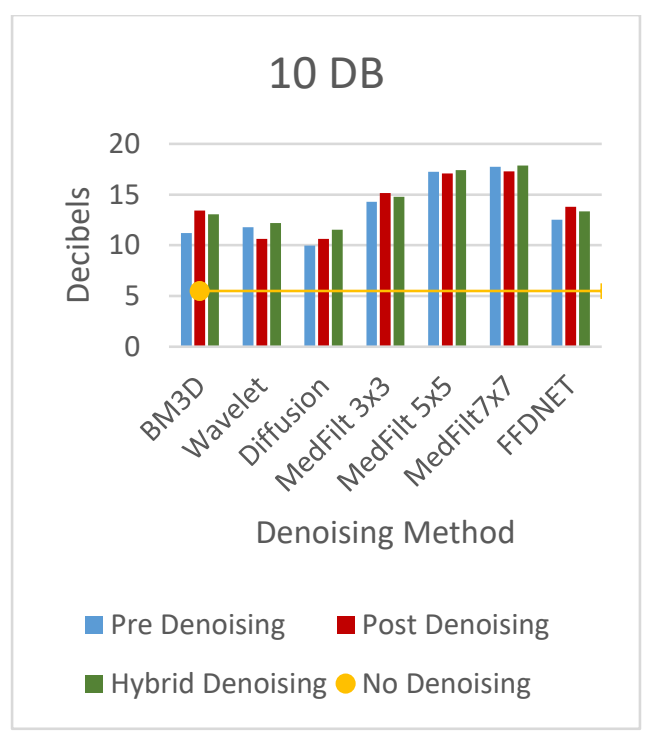

(d) HVSm

Figure 9. Comparison of different denoising methods for the $10 \mathrm{~dB}$ noisy images. 
Signal \& Image Processing: An International Journal (SIPIJ) Vol.11, No.5, October 2020

3.3.2. $20 \mathrm{~dB}$ case

For the $20 \mathrm{~dB}$ case, we have following observations:

- $\quad$ All filters improved over the no filtering case.

- $\quad$ BM3D filter has the best performance in all four metrics. It has improved the PSNR by more than $7 \mathrm{dBs}$.

- $\quad$ Wavelet, median filter with 3x3, and FFDNET have close performance.

- The worst filter is the median filter with $7 \times 7$. It appears that small filter size should be used for less noisy images.

- $\quad$ Pre-filtering is better than post-filtering in all cases except the median filter with $7 x 7$ size.

- Hybrid did not yield any gains over either pre-filtering or post-filtering.

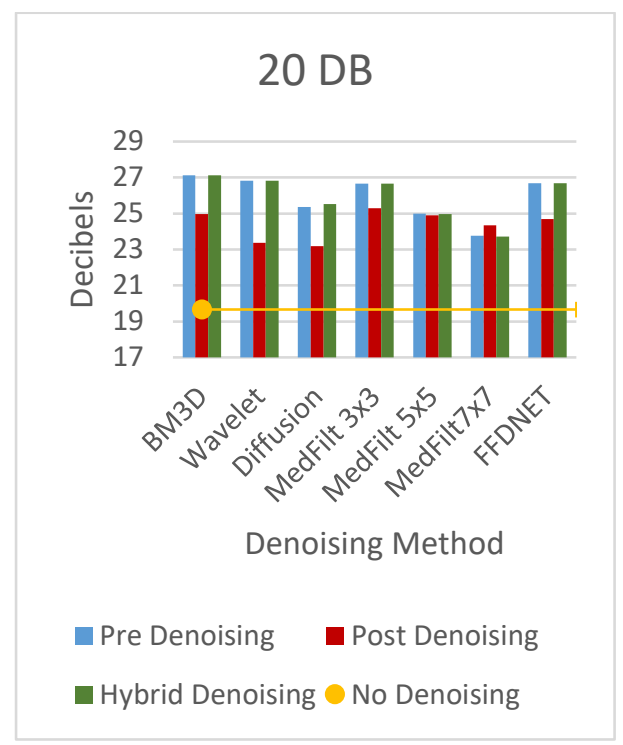

(a) PSNR

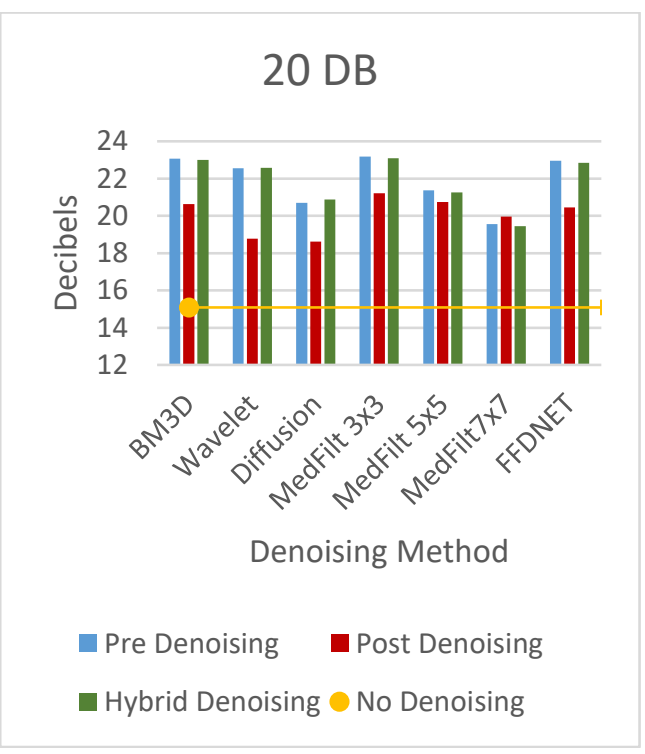

(c) HVS

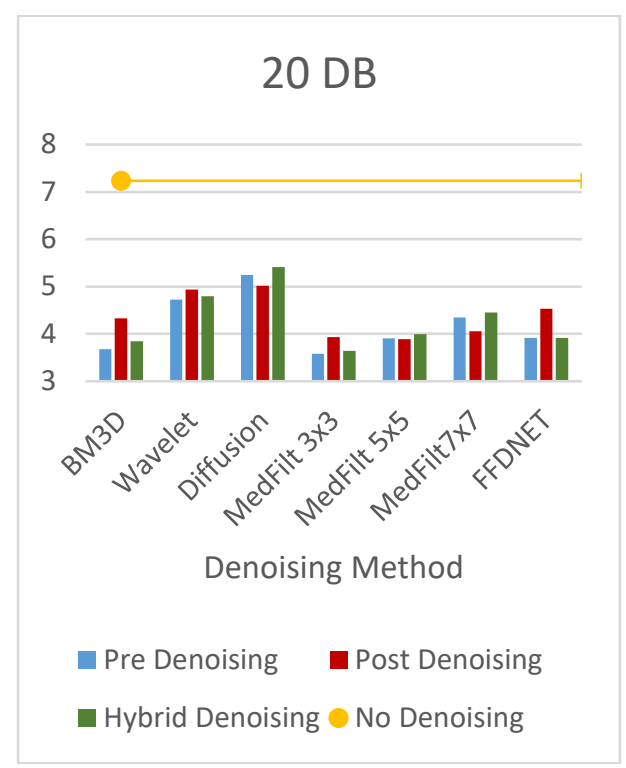

(b) CIELAB

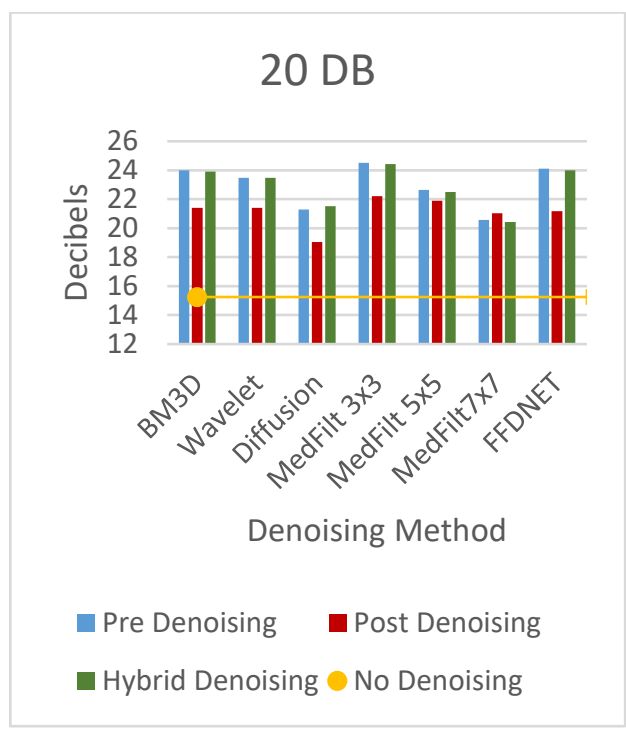

(d) HVSm

Figure 10. Comparison of different denoising methods for the $20 \mathrm{~dB}$ noisy images 
Signal \& Image Processing: An International Journal (SIPIJ) Vol.11, No.5, October 2020

\section{Conclusions}

Low light images have serious Poisson noise that affects the visual quality of images. In this paper, we present a thorough investigation of the various combination of denoising and demosaicing algorithms for low light images. Two noise levels (10 $\mathrm{dB}$ and $20 \mathrm{~dB}$ ) were investigated using six conventional and one deep learning denoising algorithms. It was observed that, in serious low lighting conditions $(10 \mathrm{~dB})$, a conventional median filter can yield better performance than more advanced algorithms whereas in mild lighting conditions $(20 \mathrm{~dB})$, some modern algorithms such as BM3D and FFDNet start to have better results. One potential future direction is to look for some better deep learning based algorithms that can specifically deal with Poisson noise.

\section{CONFLICT OF INTEREST}

The authors declare no conflict of interest.

\section{ACKNOWLEDGEMENTS}

This work was partially supported by NASA Jet Propulsion Laboratory under contract \# 80NSSC17C0035. The views, opinions and/or findings expressed are those of the author(s) and should not be interpreted as representing the official views or policies of NASA or the U.S. Government.

\section{REFERENCES}

[1] B. E. Bayer, Color imaging array. US Patent 3,971,065, July 20, 1976.

[2] J. F. Bell III, et al., "The Mars Science Laboratory Curiosity Rover Mast Camera (Mastcam) Instruments: Pre-Flight and In-Flight Calibration, Validation, and Data Archiving," AGU Journal Earth and Space Science, 2017.

[3] M. Dao, C. Kwan, B. Ayhan, and J. F. Bell III, "Enhancing Mastcam Images for Mars Rover Mission," 14th International Symposium on Neural Networks, pp. 197-206, 2017.

[4] C. Kwan, B. Budavari, M. Dao, B. Ayhan, J. F. Bell III, "Pansharpening of Mastcam images," IEEE International Geoscience and Remote Sensing Symposium, pp. 5117-5120, 2017.

[5] B. Ayhan, M. Dao, C. Kwan, H. Chen, J. F. Bell III, and R. Kidd, "A Novel Utilization of Image Registration Techniques to Process Mastcam Images in Mars Rover with Applications to Image Fusion, Pixel Clustering, and Anomaly Detection,” IEEE Journal of Selected Topics in Applied Earth Observations and Remote Sensing, 10(10), pp. 4553-4564, 2017.

[6] J. Hamilton and J. Compton, Processing color and panchromatic pixels. U.S. Patent 20070024879A1, 2007.

[7] T. Kijima, H. Nakamura, J. T. Compton, J. F. Hamilton, and T. E. DeWeese, Image sensor with improved light sensitivity. U.S. Patent 0268 533, Nov., 2007.

[8] C. Kwan and J. Larkin, "Demosaicing of Bayer and CFA 2.0 Patterns for Low Lighting Images," Electronics, 8, 1444, 2019.

[9] M. Gharbi, G. Chaurasia, S. Paris, and F. Durand, "Deep joint demosaicking and denoising," ACM Trans. Graph, 35, 2016.

[10] C. Kwan, J. Larkin, and B. Ayhan, "Demosaicing of CFA 3.0 with Application to Low Lighting Images," Sensors, 20 (12), 3423, June 22, 2020.

[11] C. Kwan, J. Larkin, and B. Budavari, "Demosaicing of Real Low Lighting Images Using CFA 3.0," Signal \& Image Processing: An International Journal (SIPIJ), vol. 11, no. 4, August 2020.

[12] C. Kwan and J. Larkin, "Demosaicing Mastcam Images Using A New Color Filter Array," Signal \& Image Processing: An International Journal, 11 (3), 2020.

[13] L. Zhang, X. Wu, A. Buades, and X. Li, "Color demosaicking by local directional interpolation and nonlocal adaptive thresholding," J. Electron. Imaging, 20, 2011.

[14] BM3D denoising: http://www.cs.tut.fi/ foi/invansc/, accessed September 22, 2020. 
Signal \& Image Processing: An International Journal (SIPIJ) Vol.11, No.5, October 2020

[15] http://cs.haifa.ac.il/hagit/courses/seminars/wavelets/Presentations/Lecture09_Denoising.pdf, accessed September 22, 2020.

[16] P. Perona and J. Malik, "Scale-space and edge detection using anisotropic diffusion," Proceedings of IEEE Computer Society Workshop on Computer Vision. pp. 16-22, November 1987.

[17] https://www.mathworks.com/help/images/ref/imdiffusefilt.html, accessed September 22, 2020.

[18] R. C. Gonzalez and R. E. Woods, Digital Image Processing, 4th Edition, 2018 Pearson, 330 Hudson Street, New York, NY 10013.

[19] K. Zhang, W. Zuo, and L. Zhang, "FFDNet: Toward a Fast and Flexible Solution for CNN based Image Denoising. arXiv:1710.04026 [cs.CV], 22 May 2018.

[20] C. Kwan, B. Chou, L. M. Kwan, and B. Budavari, "Debayering RGBW Color Filter Arrays: A Pansharpening Approach," IEEE Ubiquitous Computing, Electronics \& Mobile Communication Conference, pp. 94-100, New York City, 2017.

[21] G. Vivone, et al., "A Critical Comparison Among Pansharpening Algorithms," IEEE Trans. Geoscience and Remote Sensing, 53(5), 2015.

[22] J. G. Liu, "Smoothing filter based intensity modulation: A spectral preserve image fusion technique for improving spatial details," Int. J. Remote Sens., 21, 18, 2000.

[23] B. Aiazzi, et al., "MTF-tailored multiscale fusion of high-resolution MS and pan imagery," Photogramm. Eng. Remote Sens., 72(5), pp. 591-596, 2006.

[24] G. Vivone, et al., "Contrast and error-based fusion schemes for multispectral image pansharpening," IEEE Geosci. Remote Sensing Lett., 11(5), pp. 930-934, 2014.

[25] C. Laben and B. Brower, Process for enhancing the spatial resolution of multispectral imagery using pan-sharpening. U.S. Patent 6011 875, Jan. 4, 2000.

[26] B. Aiazzi, et al., "Improving component substitution pansharpening through multivariate regression of MS+pan data," IEEE Trans. Geosci. Remote Sensing, 45(10), pp. 3230-3239, 2007.

[27] W. Liao, et al., "Processing of multiresolution thermal hyperspectral and digital color data: Outcome of the 2014 IEEE GRSS data fusion contest," IEEE J. Select. Top. Appl. Earth Observ. Remote Sensing, 8, 6, 2015.

[28] J. Choi, et al., "A new adaptive component-substitution based satellite image fusion by using partial replacement," IEEE Trans. Geosci. Remote Sens., 49, 1, 2011.

[29] J. Zhou, C. Kwan, and B. Budavari, "Hyperspectral image super-resolution: A hybrid color mapping approach,” Journal of Applied Remote Sensing, 10, 3, article 035024, 2016.

[30] C. Kwan, J. H. Choi, S. Chan, J. Zhou, and B. Budavai, "Resolution Enhancement for Hyperspectral Images: A Super-Resolution and Fusion Approach,” IEEE International Conference on Acoustics, Speech, and Signal Processing, pp. 6180 - 6184, New Orleans, 2017.

[31] C. Kwan, B. Budavari, and F. Gao, "A Hybrid Color Mapping Approach to Fusing MODIS and Landsat Images for Forward Prediction,” Remote Sensing, 10 (4), 520, 2017.

[32] C. Kwan, B. Budavari, A. Bovik, and G. Marchisio, "Blind Quality Assessment of Fused WorldView3 Images by Using the Combinations of Pansharpening and Hypersharpening Paradigms," IEEE Geoscience and Remote Sensing Letters, 14 (10), 1835-1839, 2017.

[33] C. Kwan, B. Ayhan, and B. Budavari, "Fusion of THEMIS and TES for Accurate Mars Surface Characterization," IEEE International Geoscience and Remote Sensing Symposium, pp. 3381-3384, 2017.

[34] L. Zhang and $\mathrm{X}$. Wu, "Color demosaicking via directional linear minimum mean square-error estimation,” IEEE Trans. Image Processing, 14, 2167-2178, 2005.

[35] W. Lu and Y. P. Tan, "Color filter array demosaicking: New method and performance measures," IEEE Trans. on Image Processing, 12, 1194-1210, 2003.

[36] E. Dubois, "Frequency-domain methods for demosaicking of Bayer-sampled color images," IEEE Signal Proc. Letters, 12, 847-850, 2005.

[37] B. Gunturk, Y. Altunbasak, and R. M. Mersereau, "Color plane interpolation using alternating projections," IEEE Trans. Image Processing, 11, 997-1013, 2002.

[38] X. Wu and N. Zhang, "Primary-consistent soft-decision color demosaicking for digital cameras," IEEE Trans. Image Processing, 13, 1263-1274, 2004.

[39] C. Kwan, X. Zhu, F. Gao, B. Chou, D. Perez, J. Li, Y. Shen, and K. Koperski, "Assessment of Spatiotemporal Fusion Algorithms for Planet and Worldview Images," Sensors, 18, 1051, 2018.

[40] K. Egiazarian, J. Astola, N. Ponomarenko, V. Lukin, F. Battisti, and M. Carli, "New full quality metrics based on HVS," In Proceedings of the Second International Workshop on Video Processing and Quality Metrics, Scottsdale, AZ, USA, 22-24 January 2006. 
Signal \& Image Processing: An International Journal (SIPIJ) Vol.11, No.5, October 2020

[41] N. Ponomarenko, F. Silvestri, K. Egiazarian, M. Carli, J. Astola, and V. Lukin, “On betweencoefficient contrast masking of DCT basis functions," In Proceedings of the Third International Workshop on Video Processing and Quality Metrics for Consumer Electronics VPQM-07, Scottsdale, AZ, USA, 25-26 January 2007.

[42] X. Zhang and B. A. Wandell, "A spatial extension of cielab for digital color image reproduction,” SID Journal, 1997.

[43] C. Kwan, J. Larkin, B. Budavari, B. Chou, E. Shang, and T. D. Tran, "A comparison of compression codecs for maritime and sonar images in bandwidth constrained applications," Computers, 8 (2), 32, April 28, 2019.

[44] C. Kwan, J. Larkin, B. Budavari, E. Shang, and T. Tran, "Perceptually Lossless Compression with Error Concealment for Periscope and Sonar Videos," Signal \& Image Processing: An International Journal (SIPIJ), 10 (4), April 30, 2019.

\section{Appendix}

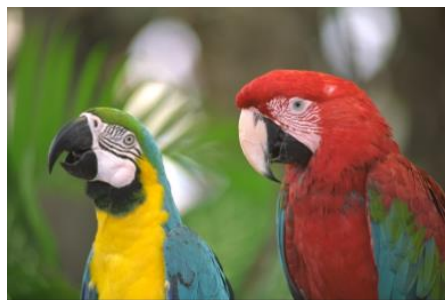

GT

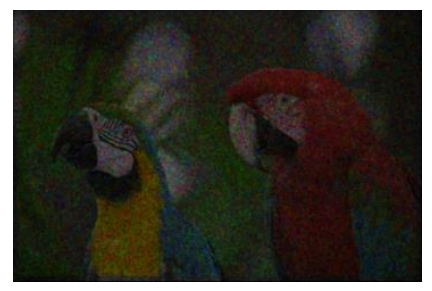

No Denoising/PRACS

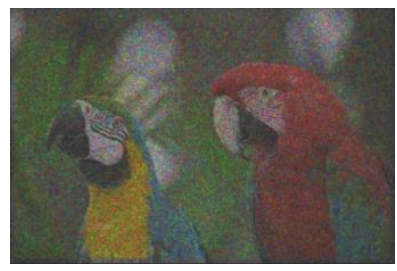

Diffusion/Standard

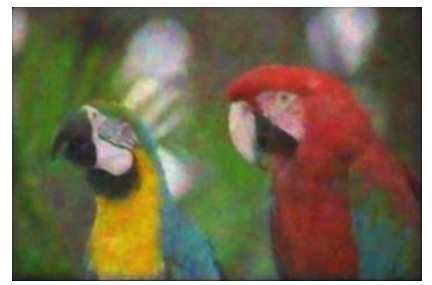

Medfilt 7x7/GFPCA

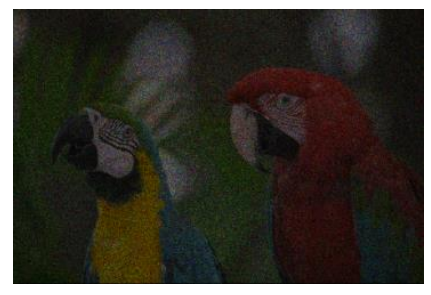

Noisy Input

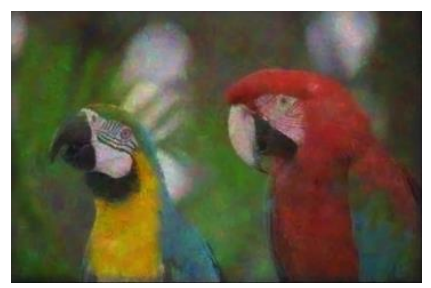

BM3D/GFPCA

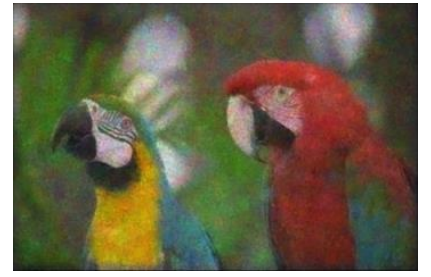

Medfilt 3x3/GFPCA

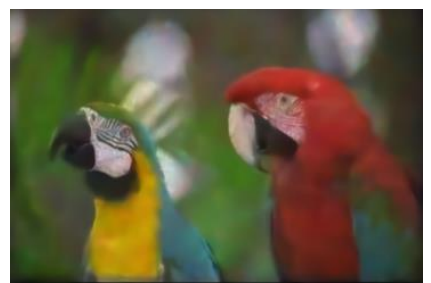

FFDNET/GFPCA

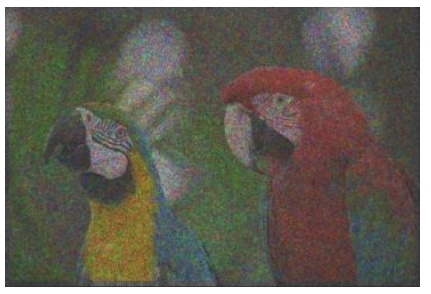

Wavelet/Standard

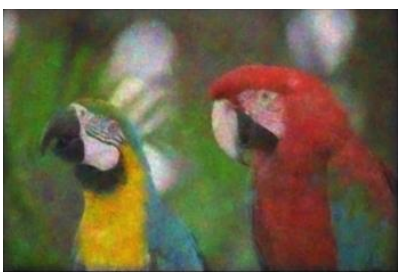

Medfilt 5x5/GFPCA

Fig. A1. Demosaicing results using various post-denoising approaches for $10 \mathrm{~dB}$ noisy images. For each image, a/b means the " $a$ " is the denoising method and " $b$ " is the pansharpening method. 
Signal \& Image Processing: An International Journal (SIPIJ) Vol.11, No.5, October 2020
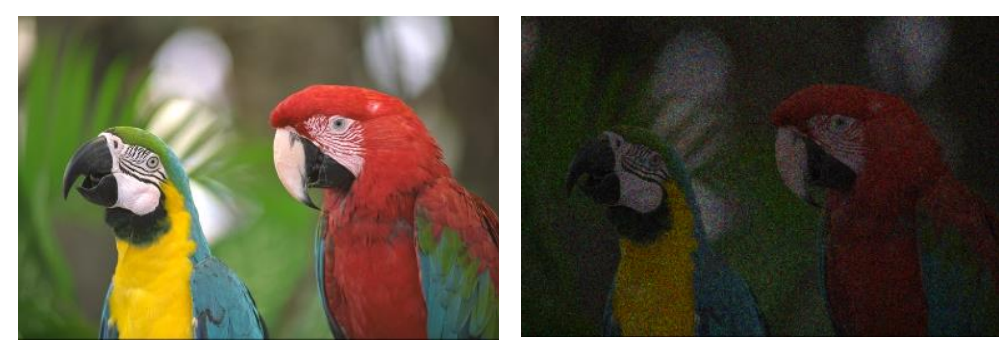

GT

Noisy Input
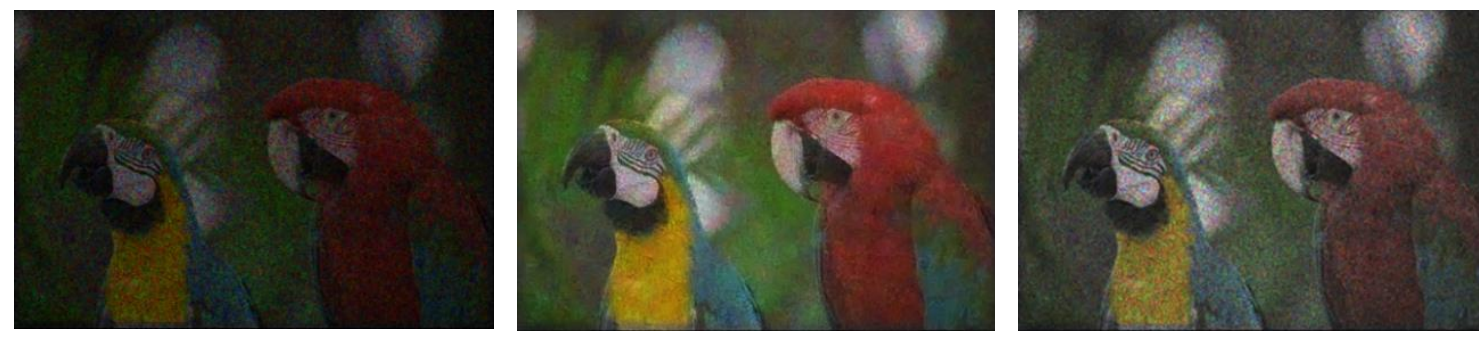

No Denoising/PRACS

BM3D/GFPCA

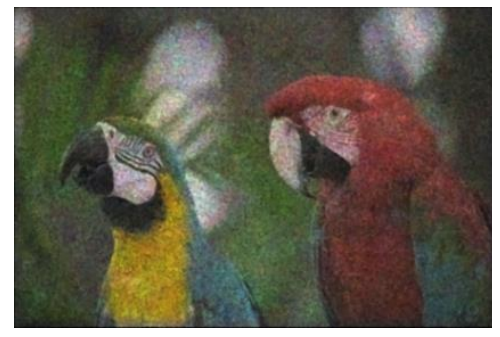

Medfilt 3x3/GFPCA

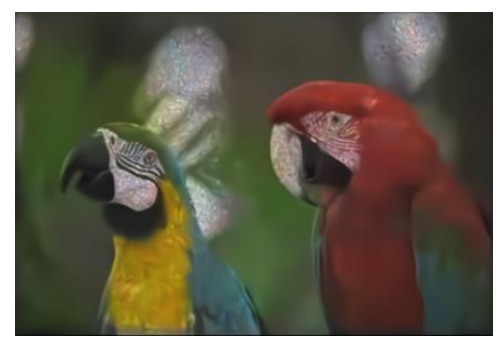

FFDNET/GFPCA
Wavelet/Baseline

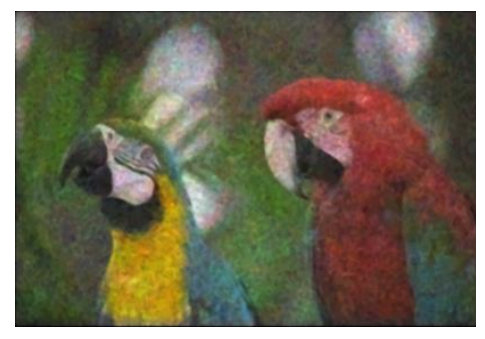

Medfilt 5x5/GFPCA

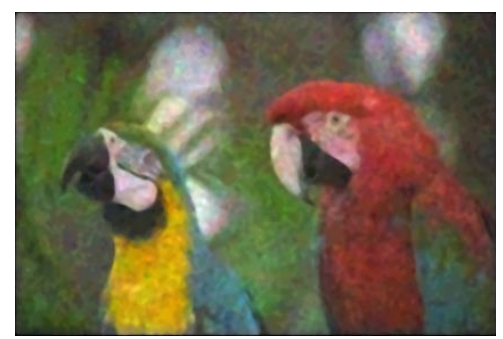

Medfilt 7x7/GFPCA

Fig. A2. Demosaicing results using various hybrid-denoising approaches for $10 \mathrm{~dB}$ noisy images. For each image, $a / b$ means the " $a$ " is the denoising method and " $b$ " is the pansharpening method. 
Signal \& Image Processing: An International Journal (SIPIJ) Vol.11, No.5, October 2020

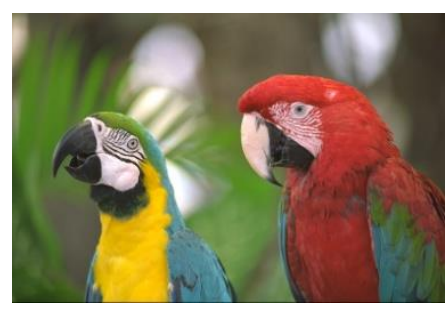

GT

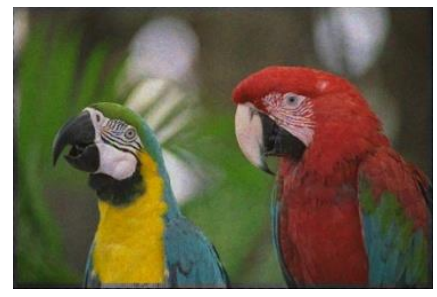

No Denoising/Standard

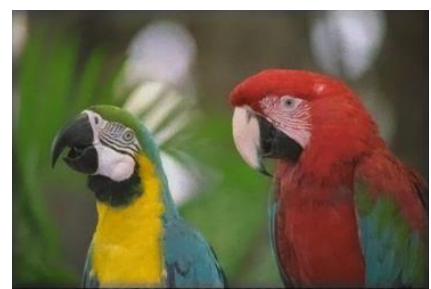

Diffusion/GFPCA

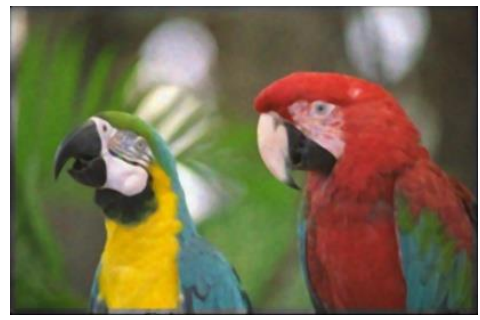

Medfilt 7x7/Baseline

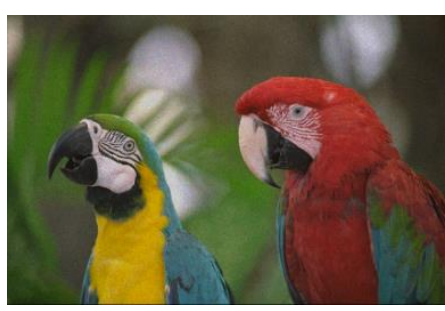

Noisy Input

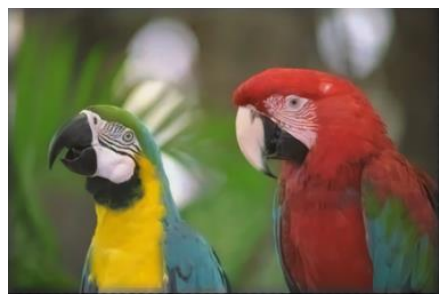

BM3D/GFPCA

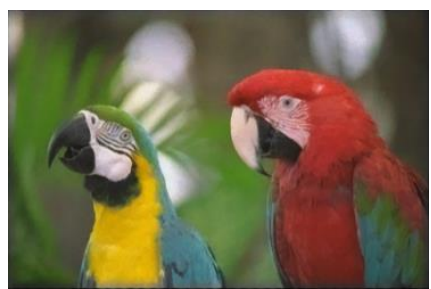

Medfilt 3x3/GFPCA

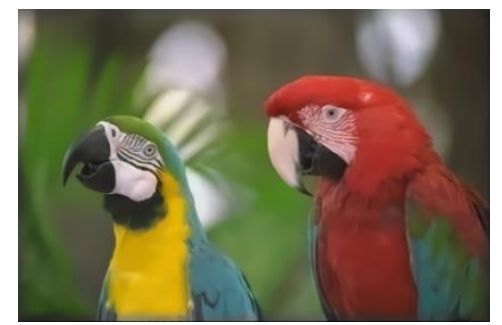

FFDNET/GFPCA

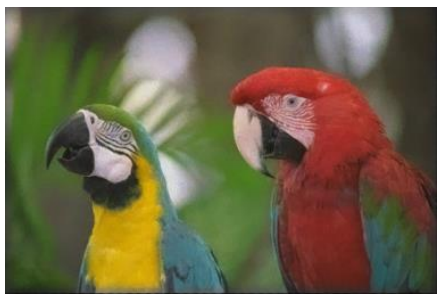

Wavelet/GFPCA

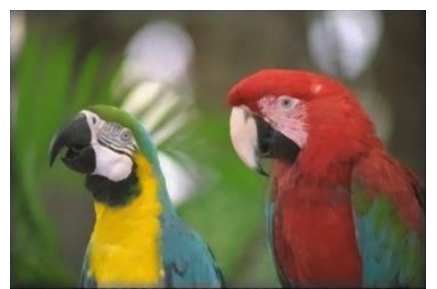

Medfilt 5x5/GFPCA

Fig. A3. Demosaicing results using various post-denoising approaches for $20 \mathrm{~dB}$ noisy images. For each image, a/b means the "a" is the denoising method and " $b$ " is the pansharpening method. 
Signal \& Image Processing: An International Journal (SIPIJ) Vol.11, No.5, October 2020

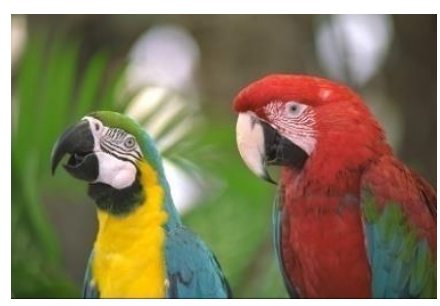

GT

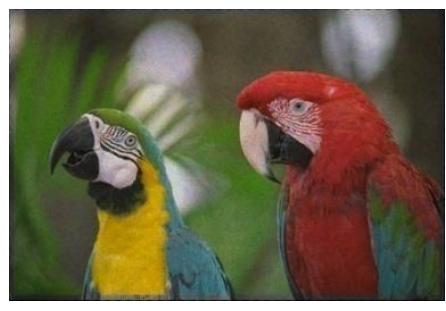

No Denoising/Standard

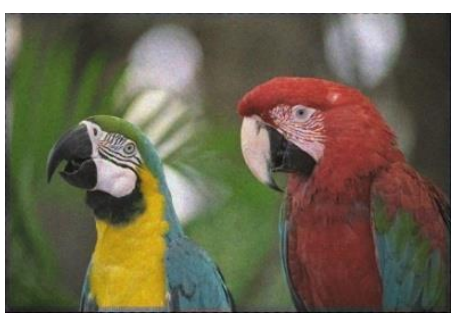

Diffusion/GSA

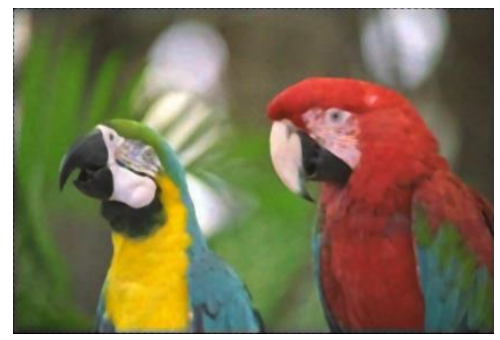

Medfilt 7x7/Standard

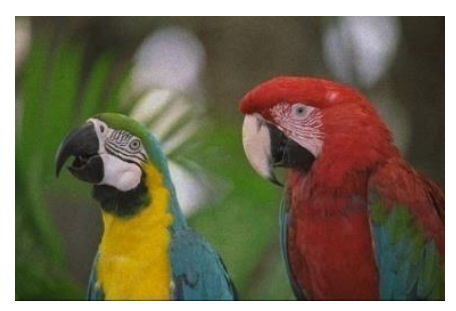

Noisy Input

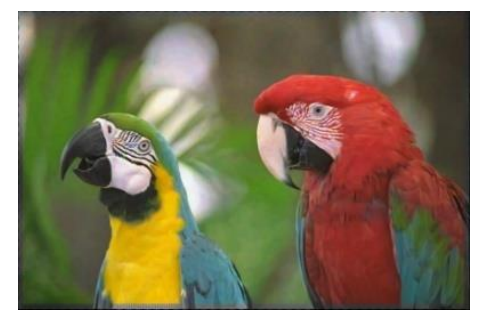

BM3D/Standard

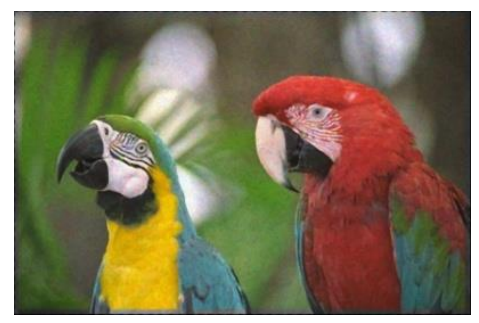

Medfilt 3x3/Standard

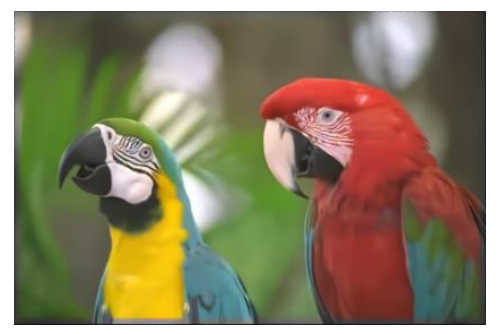

FFDNET/Standard

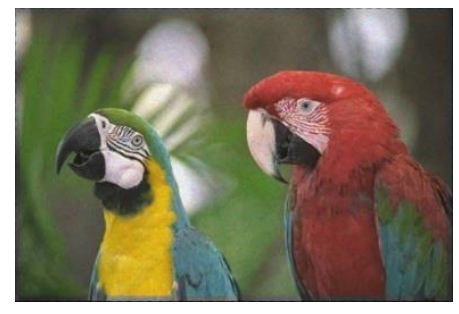

Wavelet/Standard

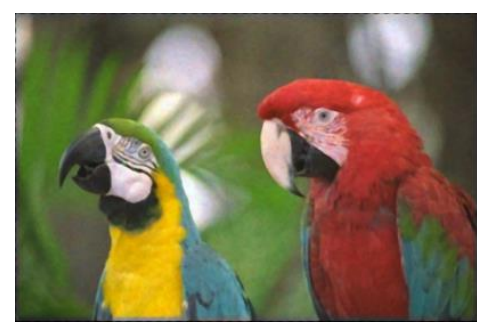

Medfilt 5x5/Standard

Fig. A4. Demosaicing results using various hybrid-denoising approaches for $20 \mathrm{~dB}$ noisy images. For each image, $a / b$ means the " $a$ " is the denoising method and " $b$ " is the pansharpening method.

\section{AuTHORS}

Chiman Kwan received his Ph.D. degree in electrical engineering from the University of Texas at Arlington in 1993. He has one book, four book chapters, 15 patents, 65 invention disclosures, 375 technical papers in journals and conferences, and 550 technical reports. Over the past 25 years, he has been the PI/Program Manager of over 120 diverse projects with total funding exceeding 36 million dollars. He is also the founder and Chief Technology Officer of Signal Processing, Inc. and Applied Research LLC. He received numerous awards from IEEE, NASA, and some other agencies.

Jude Larkin received his B.S. in Computer Science from Franciscan University of Steubenville in 2015. $\mathrm{He}$ is a software engineer at ARLLC. He has been involved in diverse projects, including mission planning for UAVs, image fusion, image demosaicing, and remote sensing. 